\title{
Targeting Lactate Dehydrogenase B-dependent Mitochondrial Metabolism Affects Tumor Initiating Cells and Inhibits Tumorigenesis of Non-small Cell Lung Cancer by Inducing MtDNA Damage
}

Thomas Michael Marti ( $\square$ Thomas.Marti@insel.ch )

University of Bern: Universitat Bern https://orcid.org/0000-0003-3005-220X

Haibin Deng

University of Bern: Universitat Bern

Yanyun Gao

University of Bern: Universitat Bern

Verdiana Trappetti

University of Bern: Universitat Bern

Damian Hertig

University of Bern: Universitat Bern

Darya Karatkevich

University of Bern: Universitat Bern

Tereza Losmanova

University of Berne Institute of Pathology: Universitat Bern Institut fur Pathologie

Christian Urzi

University of Bern: Universitat Bern

Huixiang Ge

University of Bern: Universitat Bern

Gerrit Adriaan Geest

University of Bern: Universitat Bern

Remy Bruggmann

University of Bern: Universitat Bern

Valentin Djonov

University of Bern: Universitat Bern

Jean-Marc Nuoffer

University of Bern: Universitat Bern

Peter Vermathen

University of Bern: Universitat Bern

Nicola Zamboni

ETH Zürich D-BIOL: Eidgenossische Technische Hochschule Zurich Departement Biologie 
Carsten Riether

University of Bern: Universitat Bern

Adrian Ochsenbein

University of Bern: Universitat Bern

Ren-Wang Peng

University of Bern: Universitat Bern

Gregor Kocher

University of Bern: Universitat Bern

Ralph Alexander Schmid

University of Bern: Universitat Bern

Patrick Dorn

University of Bern: Universitat Bern

\section{Research Article}

Keywords: Lung cancer, Cancer stem cells, tumorigenicity, mitochondrial metabolism, cellular plasticity, mitochondrial DNA, nucleotide metabolism

Posted Date: January 24th, 2022

DOI: https://doi.org/10.21203/rs.3.rs-1271874/v1

License: (c) (i) This work is licensed under a Creative Commons Attribution 4.0 International License. Read Full License 


\section{Abstract}

Once considered a waste product of anaerobic cellular metabolism, lactate has been identified as a critical regulator of tumorigenesis, maintenance, and progression. The putative primary function of lactate dehydrogenase $B(\mathrm{LDHB})$ is to catalyze the conversion of lactate to pyruvate; however, its role in regulating metabolism during tumorigenesis is largely unknown. To determine whether LDHB plays a pivotal role in tumorigenesis, we performed $2 \mathrm{D}$ and $3 \mathrm{D}$ in vitro experiments, utilized a conventional xenograft tumor model, and developed a novel genetically engineered mouse model (GEMM) of nonsmall cell lung cancer (NSCLC), in which we combined an $\angle D H B$ deletion allele with an inducible model of lung adenocarcinoma driven by the concomitant loss of $p 53$ (also known as Trp53) and expression of oncogenic KRAS (G12D) mutant (KP). Here, we show that epithelial-like, tumor-initiating NSCLC cells feature oxidative phosphorylation (OXPHOS) phenotype that is regulated by LDHB-mediated lactate metabolism. We show that silencing of $\angle D H B$ induces persistent mitochondrial DNA damage, decreases mitochondrial respiratory complex activity and OXPHOS, resulting in reduced levels of mitochondriadependent metabolites, e.g., TCA intermediates, amino acids, and nucleotides. In addition, inhibition of LDHB dramatically reduced the survival of tumor-initiating cells and sphere formation in vitro, which can be partially restored by nucleotide supplementation. In addition, $L D H B$ silencing reduced tumor initiation and growth of xenograft tumors. Furthermore, we report for the first time that homozygous deletion of $\angle D H B$ significantly reduced lung tumorigenesis upon the concomitant loss of $T p 53$ and expression of oncogenic KRAS without considerably affecting the animal's health status, thereby identifying LDHB as a potential target for NSCLC therapy. In conclusion, our study shows for the first time that LDHB is essential for the maintenance of mitochondrial metabolism, especially nucleotide metabolism, demonstrating that LDHB is crucial for the survival and proliferation of tumor-initiating cells and tumorigenesis.

\section{Background}

The tumor is a complex system containing a minority of highly tumorigenic cells ( $<1 \%$ in the majority of solid tumors), referred to as cancer stem cells or tumor-initiating cells (TICs). TICs have been discovered in many solid tumors, including breast, brain, skin, lung, pancreatic, and colon (1-6). TICs are thought to contribute to the tumor initiation, maintenance, progression, resistance to treatments, and recurrence or metastasis of cancer (7-10). In lung cancer, TICs have been identified using several markers, e.g., drugresistant side-population, $\mathrm{CD} 133^{+}, \mathrm{ALDH}^{\text {high }}$, and $\mathrm{EpCAM}^{+}$. Recently, glycine decarboxylase (GLDC) was identified as the most robust TIC marker in NSCLC (11). Intriguingly, GLDC is mainly expressed in the mitochondria $(12,13)$. A high level of oxidative phosphorylation (OPXHOS) correlates with increased sphere formation and tumor growth capacity in NSCLC (14). In addition, a recent study has shown that a functional OXPHOS is critical for tumorigenesis. Specifically, loss of mitochondria-dependent pyrimidine synthesis rather than mitochondria-dependent ATP production is responsible for the loss of tumorigenic ability (15). Indeed, loss of mitochondria-dependent nucleotide synthesis is synthetically lethal in cancers induced by a KRAS mutation (16). Although considered a waste product of anaerobic glycolysis (e.g., Warburg effect), lactate has been shown to serve as a source of respiration in cancer, and targeted 
lactate-fueled respiration can selectively kill tumor cells in mice (15). More recently, research revealed that lactate is the primary carbon source for the TCA cycle in vivo, providing substrate and electrons for oxidative phosphorylation (OXPHOS) in normal tissue and lung tumors $(16,17)$. Thus, lactate drives mitochondrial metabolism, but its relationship to TIC survival is unclear.

The tetrameric enzyme lactate dehydrogenase (LDH), encoded by the genes lactate dehydrogenase $A$ and $\mathrm{B}$ ( $\angle D H A$ and $\angle D H B$, respectively), catalyzes the interconversion of pyruvate and lactate using $\mathrm{NADH} / \mathrm{NAD}^{+}$as a co-substrate, and which is encoded by the genes lactate dehydrogenase $\mathrm{A}$ and $\mathrm{B}$ ( $L D H A$ and $\angle D H B$, respectively) (reviewed in (18)). LDHA, particularly the LDHA-homo-tetramer, converts pyruvate to lactate, whereas LDHB primarily converts lactate to pyruvate. Only the double-knockout of $L D H A$ and $\angle D H B$ entirely suppressed LDH activity and lactate secretion (19). Thus, LDHB may at least partially replace the function of LDHA in promoting glycolysis. LDHA has been shown to be essential for tumorigenesis, growth, and progression of different cancers (reviewed in $(18,20,21))$. Furthermore, LDHA abrogation has been shown to reduce tumorigenesis and tumor growth and decrease the survival and proliferation of TICs in a novel inducible NSCLC mouse models in the context of KRAS or EGFR mutations (22). Interestingly, abrogation of LDHA results not only in inhibition of glycolysis but also in metabolic reprogramming leading to enhanced mitochondrial metabolism (22), suggesting that LDHA expression is essential for glycolysis but may not be critical for maintaining mitochondrial function. However, LDHB is localized to the mitochondria and treatment with an LDH inhibitor decreased respiration of isolated mitochondria cultured in lactate but not in pyruvate (23). Taken together, LDHB may be more related to mitochondrial metabolism and its inhibition may even have an opposite effect on mitochondrial metabolism compared to LDHA. The relationship between LDHB expression and cancer is complex: LDHB is silenced by promoter methylation in several cancers, but increased LDHB expression has been described in several adenocarcinomas, including NSCLC (24). Increased LDHB expression is associated with poor survival in many cancers $(25,26)$. In particular, LDHB has been shown to be required for the growth of KRASmutated lung cancer cells in vitro and in vivo (27) and to play a critical role in hyperactive mTOR-mediated tumorigenesis (28). However, whether TICs of NSCLC rely on high levels of OXPHOS, whether LDHB is essential for lactate-mediated OXPHOS for TICs, and especially how LDHB regulates mitochondrial metabolism, remains largely unknown.

Our current study demonstrated that TICs in NSCLC are characterized by an OXPHOS phenotype, which relies on LDHB activity. Inhibition of LDHB induces persistence of mtDNA damage and dramatically reduces OXPHOS and mitochondrial metabolism-dependent nucleotide metabolism leading to a reduction in survival of TICs in NSCLC. Our study revealed that LDHB has a vital role in tumorigenesis, both mouse xenograft tumor models and GEMMs. Thus, our research revealed that LDHB is essential to maintain mitochondrial DNA integrity and mitochondrial metabolism, which is crucial for tumor-initiating cells and tumorigenesis.

\section{Results}




\section{TICs are characterized as OXPHOS phenotype and tend to lactate utilization}

To better understand the metabolic synergy between lung cancer cells, we extended our characterization of subpopulations in the NSCLC cell line A549, where the mesenchymal paraclone subpopulation (PARA) had the lowest tumor-initiating capacity, whereas the epithelial holoclone subpopulation (HOLO) had the highest tumor-initiating capacity (29). We focused on lactate metabolism, which fuels lung cancer metabolism (17). Reanalysis of our previously published data (29) revealed that hexokinase 2 (HK2), LDHA, and the sodium lactate transporter MCT4 (encoded by SLC16A3) catalyzing the first step of glucose metabolism, lactate synthesis, and lactate secretion, respectively, are significantly overexpressed in PARA compared to the HOLO (Supplementary Fig. S1a). In contrast, LDHB, which mainly converts lactate and $\mathrm{NAD}^{+}$to pyruvate and $\mathrm{NADH}$, is expressed at higher levels in HOLO (Supplementary Fig. S1a). Therefore, we postulated a simple model in which A549 paraclonal cells process glucose by glycolysis and secrete lactate, which drives energy metabolism in holoclonal cells. In agreement with this model, the glucose-dependent extracellular acidification rate (ECAR), which is associated with glycolysis, is increased in paraclonal A549 cells. In contrast, the basal oxygen consumption rate (OCR) is decreased compared to holoclonal cells (Fig. 1a and Supplementary Fig. S1b). Compared to paraclonal cells, the decrease in basal OCR of holoclonal cells was significantly more pronounced after treatment with oligomycin which is an inhibitor of ATP synthase, indicating that the ATP production in holoclonal cells is more dependent on OXPHOS compared to paraclonal cells (Fig. 1a and Supplementary Fig. S1c). Indeed, the hallmarks of functional OXPHOS, e.g., maximal respiration and spare respiration, were also significantly increased in holoclonal A549 cells (Fig. 1a and Supplementary Fig. S1c). Interestingly, although glycolysis and glycolytic capacity were increased compared with holoclonal cells, the glycolytic reserve was negligible in paraclonal cells, suggesting that glycolysis in paraclonal cells runs at nearly full capacity (Supplementary Fig. S1d).

Moreover, lactate supplementation specifically increased the OCR of holoclonal cells (Fig. 1b) and rescued the survival of holoclonal cells under glucose deprivation (Fig. 1c). Consistent with this, lactate supplementation also increased in the A549 and H358 cell lines the proportion of the subpopulations characterized by elevated GLDC, Aldehyde dehydrogenase (ALDH) activity and SOX2 expression, critical markers associated with heightened tumor initiation capacity $(11,30)$, also increased in A549 or H358, respectively (Fig. 1d and Supplementary Fig. S1e).

Thus, TICs in NSCLC have increased OXPHOS levels and prefer lactate utilization compared with nonTICs.

\section{LDHB mediated lactate utilization is critical to OXPHOS}

To further dissect how LDHB-mediated lactate metabolism affects TICs, we performed a short-term inhibition of LDHB expression by using siRNA, which significantly reduced LDHB expression and activity. As a negative control, MCF7 breast cancer cells that do not express LDHB showed no detectable LDHB 
activity assay (Fig. 2a-b and Supplementary Fig. S2a). Given that LDHB mainly catalyzes the conversion of lactate to pyruvate, we first investigated whether LDHB activity is important for lactate utilization. We performed rescue experiments, e.g., we determined the effect of lactate supplementation on the growth of cancer cells cultured in a medium without glucose and glutamine. Lactate supplementation was able to rescue the growth of parental A549 cells but not after LDHB silencing (Fig. 2c). Further, we also determined the uptake of ${ }^{13} \mathrm{C}$-labelled lactate by NMR as described before (37). Silencing LDHB reduced lactate uptake from serum in A549 cells under low glucose conditions (Fig. 2d), suggesting that LDHB activity is required for lactate uptake. Since previous research indicated that lactate can be used as fuel for mitochondrial respiration and lactate is the primary fuel for the TCA cycle $(15,31)$, we speculated that lactate utilization mediated by LDHB contributes to OXPHOS. Indeed, acute silencing of LDHB significantly reduced basal, ATP-linked, and maximal OCR in a panel of NSCLC cell lines and the primary culture PF139 (Fig. 2e and Supplementary Fig. S2b-c). To better understand whether inhibition of LDHB after long-term adaptation reduces OXPHOS, we also generated shRNA-mediated LDHB silencing cells that exhibit dramatically reduced LDHB expression and activity (Figs. 2f-g). Not surprisingly, basal, ATPlinked, and maximal OCR were significantly decreased in A549 cells that stably expressed LDHB-specific shRNAs. (Fig. 2h). These results indicate that LDHB is essential for functional OXPHOS in lung cancer cells.

It was shown before that inhibition of LDHA by a small molecule inhibitor decreases glycolysis and leads to a compensatory increase in OXPHOS (32). Therefore, we speculated that silencing LDHB would increase ECAR. Surprisingly, silencing of LDHB decreased ECAR, i.e., a hallmark of glycolysis, in all tested cell lines (Fig. S2d). In addition, glycolytic capacity and reserve were also reduced after LDHB silencing (supplemental Figure S2e), suggesting that the alteration in metabolism induced by LDHB inhibition is not identical to LDHA inhibition. In summary, silencing of LDHB reduced OCR and ECAR capacity, which was associated with a shift from an energetic to a more quiescent phenotype (Supplementary Fig. S2f).

\section{LDHB inhibition results in reduced survival of TICs, tumor initiating capacity and growth}

It has been shown that TICs are dependent on functional OXPHOS (33). Indeed, the level of OXPHOS in A549 holoclonal cells was increased compared to paraclonal A549 cells (see Fig. 1a). Further, silencing of LDHB significantly reduced OXPHOS, e.g., basal, maximal, and ATP-linked OCR in a panel of NSCLC cell lines and the primary culture PF139 (Fig. 2e and Supplementary Fig. S2b-c). Thus, we speculated that inhibition of LDHB should influence TICs through decreasing OXPHOS. Indeed, the fraction of holoclonal cells was decreased in the parental A549 cell line upon LDHB silencing (Fig. 3a). Interestingly, the fraction of paraclonal cells was increased upon LDHB silencing (Fig. 3a and Supplementary Fig. S3a). To assess whether LDHB inhibition is preferred by non-TICs, we then performed LDHB silencing in purified cultures from holoclone and paraclone separately. We found that LDHB silencing reduced not only the proliferation of holoclone cells but also paraclonal cells (Supplementary Fig. S3b, left panel). The relative resistance to LDHB inhibition of the purified paraclonal culture was increased compared to the holoclonal 
culture (Supplementary Fig. S1b, right panel), indicating that the holoclonal status, which is associated with increased tumor initiation capacity, is highly sensitive to LDHB inhibition. Further, silencing of LDHB not only reduced the fraction of holoclonal 4549 cells but also reduced the fraction of $\mathrm{GLDC}^{+}$cells in the A549, H358 cell lines (Fig. 3b and Fig. S3c). LDHB silencing also reduced the fraction of $\mathrm{ALDH}^{+}$cells in the A549, H358, and $\mathrm{H} 460$ cell lines (Fig. $3 \mathrm{c}$ and Fig. S3d).

In the NSCLC cell lines A549, H358, H460, H838, and the primary culture PF139, silencing of LDHB dramatically reduced colony and sphere formation, which serves as an in vitro surrogate assay to assess tumor-initiation $(34,35)$ (Fig. 3d and Fig.S3e). Since LDHB is part of the KRAS amplicon (27), we speculated that the sensitivity to LDHB silencing might be limited to KRAS mutated cell lines. However, LDHB silencing also reduced sphere and colony formation of the NSCLC cell lines H520 and H1650, both of which harbor a wild-type form of KRAS (Supplementary Fig. S3e). Additionally, in silico analysis of the lung adenocarcinoma patient's samples available in the TCGA database revealed that LDHB expression correlated with the TP53 mutational status (Fig. S3f). Thus, it is unlikely that sensitivity to LDHB inhibition depends solely on the mutational status of KRAS. Subsequently, the effect of long-term LDHB inhibition on TICs was also investigated. The proportion of TIC-like cells, i.e., holoclonal cells as well as cells characterized by increased GLDC expression, remained lower after long-term adaptation to LDHB silencing compared with A549 shCTRL cells (Fig. 3e and Supplementary Fig. S3g), despite the significant heterogeneity in EMT status among the A549 shLDHB clones examined (Fig. S3g). Further, the sphere and colony formation of shLDHB cells continued to be suppressed (Fig. $3 f$ and Supplementary Fig. S3h).

Strikingly, the tumor initiation capacity of the shLDHB-1 and shLDHB-2 clones was reduced by 62 - and 253-fold compared to the A549 shCTRL clones (Fig. 3g). In addition, after implantation of A549 shLDHB cells, the growth rate of the few initiated tumors remained markedly reduced compared with the control tumors (Fig. 3h). Similarly, in the few tumors initiated after implantation of A549 shLDHB cells, LDHB expression remained generally reduced compared with control tumors. However, although both LDHB and GLDC expression levels varied significantly between tumor regions (Supplementary Fig. S3i).

In addition, the proliferation of $\mathrm{A} 549, \mathrm{H} 358, \mathrm{H} 460$, and primary culture PF139 was also dramatically reduced after LDHB silencing (Supplementary Fig. S3j). Interestingly, in the cell line H838, which features the highest LDHB expression levels of the 135 NSCLC cell lines included in the cBioportal database, silencing of LDHB reduced sphere and colony formation, whereas proliferation was not affected (Fig. 3d and Supplementary Fig. S3e, j). These results suggest that different metabolic dependencies might characterize proliferation and tumorigenesis. Finally, silencing of LDHB did not significantly reduce proliferation and colony formation of the human non-tumorigenic lung epithelial cell line BEAS-2B (Supplementary Fig. S3k), which is consistent with a previous study showing that LDHB silencing reduces proliferation of several cancers but not normal cell lines (36). Together, these results suggest an essential role for LDHB in tumorigenesis and growth that may be cancer-specific.

\section{OXPHOS deficiency induced by LDHB inhibition is related to persistent mtDNA damage}


To investigate the molecular mechanisms underlying the pleiotropic cellular changes induced by LDHB silencing, we performed a comprehensive whole transcriptome expression analysis of A549 and H358 cell lines. Silencing LDHB significantly reduced the expression of 1789 genes in both A549 and the H358 cells (Fig. 4a, left panel), which enriched in the pathways associated with the mitochondrial morphology, e.g., mitochondrial envelope and mitochondrial matrix (Fig. 4a, right panel), which was in agreement with the co-localization of LDHB with mitochondria in A549 cells (Supplementary Fig. S4a) and a previous report in HeLa cells (23). Gene set enrichment analysis (GSEA) based on Hallmark gene sets also shows that the oxidative phosphorylation pathway is significantly enriched in scramble control cells compared with LDHB-silencing cells in A549 and H358 cell lines (Supplementary Fig. S4b), consistent with our results showing that LDHB silencing inhibits OXPHOS. These analyses indicate that LDHB is important for mitochondrial function.

The electron transport chain (ETC) of the mitochondrial oxidative phosphorylation system consists of $\sim 80$ polypeptides, most of which are nuclear-encoded, except for the 13 core subunits encoded by mitochondrial DNA, which additionally encodes two ribosomal RNAs and 22 transfer RNAs necessary for translation of the 13 proteins (37). Our GSEA based on the HUGO Gene Nomenclature Committee (HGNC) mitochondrial respiratory chain complex gene group revealed that genes related to mitochondrial respiratory chain complexes were significantly enriched in scramble control cells compared with LDHBsilencing cells in A549 and H358 cell lines (Fig. 4b). This analysis suggests that LDHB silencing reduces the expression of both nuclear and mitochondrial DNA-encoded mitochondrial genes, consistent with the reduced OXPHOS of the cancer cell lines tested (see Fig. 2). Intriguingly, the expression of CMPK2, which provides nucleotides for mtDNA repair and replication (38), is among the genes whose expression increased most upon LDHB silencing in both the A549 and the H358 cell lines (expression increased more than 6-fold, see supplementary Excel file Gene Expression). We, therefore, hypothesized that OXPHOS deficiency induced by silencing LDHB was associated with impairment of mitochondrial DNA integrity. Indeed, silencing of LDHB decreased the relative amplification of mtDNA in the A549 and H358 cell lines and also in shRNA long-term adaptation cells (Fig. 4C), suggesting that silencing of LDHB induces mtDNA damage leading to decreased mtDNA expression. Indeed, the activity of individual mitochondrial ETC complexes I and IV, encoded by mitochondrial DNA, and complex II, encoded by nuclear DNA, was reduced after short-term silencing of LDHB and remained reduced regardless of substrate availability during long-term adaptation (Fig. 4d-e and supplemental Fig. S4c-d), indicating that the OXPHOS capacity per se is reduced independent of the supply of the TCA-derived electron carrier fueling OXPHOS, e.g., NADH. In this context, it was shown that both NAD ${ }^{+}$and NADH levels were dramatically increased in OXPHOS-deficient Rho 0 cells upon pyruvate starvation (39). Indeed, both NAD+ and NADH levels were significantly increased after long-term LDHB silencing (Supplementary Fig. S4e).

Thus, these findings further support our hypothesis that persistent mitochondrial DNA damage induced by LDHB silencing might be the underlying molecular feature responsible for the inability of a cancer cell 
to adapt their mitochondrial-encoded OXPHOS and consequently their mitochondria-dependent metabolism, thereby abolishing the tumor initiation capacity.

\section{LDHB inhibition downregulates mitochondrial-dependent pyrimidine and purine synthesis pathways}

It is well established that functional mitochondria are essential for nucleotide metabolism, which is important for tumorigenesis (40-44). Therefore, we performed metabolomics analysis through an untargeted liquid chromatography-mass spectrometry (LC-MS) to corroborate our gene expression analysis data and provide additional unbiased and comprehensive insights into how LDHB silencing affects cancer cells and whether LDHB links mitochondrial metabolism to nucleotide metabolism. In agreement with the data of our gene expression analysis, levels of metabolites and genes related to the mitochondrial-dependent metabolism were generally reduced after silencing of LDHB, e.g., TCA cycle and amino acid (Fig. 5b and Supplementary Fig. S5a-b), supporting our conclusion that silencing of LDHB targets mitochondria-related metabolism.

In both A549 and H358 cell lines, silencing of LDHB significantly reduced 73 metabolites that are significantly enriched in the nucleotide metabolic pathway, such as pyrimidine and purine metabolism (Fig. 5a), indicating that silencing LDHB reduces nucleotide metabolism. In detail, the level of nucleotides associated with pyrimidine metabolism, such as UDP, UMP, UTP, CTP, CDP, dTTP, reduced dramatically, and also glutamine level which is a substrate for pyrimidine synthesis (Fig. $5 \mathrm{~b}$ and Supplementary Fig. S5c). Meanwhile, the level of nucleotides and substrates associated with purine metabolism, such as IMP, AMP, ADP, GDP, 5-phosphoribosylamine, and FGAR, also reduced dramatically upon LDHB silencing (Fig. $5 \mathrm{~b}$ and Supplementary Fig. S5c). In addition, metabolites associated with mitochondria-dependent onecarbon and methionine cycle metabolism, which are important for nucleotide synthesis, are also reduced (Fig. 5b). Our in silico analysis of data previously published by Quin et al. (45) showed that reduction of LDHB expression by treatment with R-2HG or with siRNA, as performed in our current study, resulted in nearly identical depletion of metabolites (Supplementary Fig. S5d left panel). Indeed, out of the 45 metabolites depleted after LDHB silencing, 30 were also depleted after R-2HG treatment. Those metabolites were most significantly related to pyrimidine and purine metabolism, e.g., nucleotide synthesis (Supplementary Fig. S5d right panel). This analysis further validates our conclusion that LDHB inhibition results in reduced mitochondria-dependent biogenesis, especially pyrimidine and purine metabolism.

To verify whether reduced nucleotide is responsible for inhibited tumor initiating capacity and proliferation, we subsequently performed a rescue experiment by supplementation with nucleotide precursors, e.g., hypoxanthine, uridine, adenine. The addition of exogenous nucleotide precursors did not increase the maximal proliferation of siCTRL-transfected A549 cells but did significantly increase the reduced proliferation rate after LDHB silencing. (Fig. 5c and Supplementary Fig. S5e). Indeed, nucleotide supplementation also partially rescued sphere formation upon LDHB silencing in A549 cells (Fig. 5d). 


\section{Depletion of LDHB results in decreased tumorigenesis and delayed tumor growth in a genetically engineered mouse model of NSCLC}

In contrast to a conventional mouse xenograft tumor model, GEMMs enable the initiation of orthotopic tumors that best mimic the pathological conditions of human tumors in terms of immune compatibility and tumor microenvironment (46). Therefore, to better understand the role of LDHB in NSCLC tumorigenesis, we developed a preclinical GEMM of NSCLC in which lung tumors can be induced by induction of KRAS mutation and TP53 deletion in addition to LDHB depletion or wild-type status, e.g.,

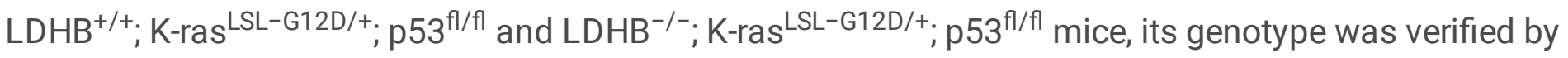
Southern blot (Fig. 6a-C, Supplementary Fig. S6a). A complete hereditary deficiency of LDHB expression was identified in two patients (47). Further, the homozygous deletion of LDHB in the mouse model results only in a minor phenotype, e.g., increased lean body mass, decreased total body fat/circulating insulin, but no immunodeficiency was observed (www.mousephenotype.org). Therefore, we speculated that LDHB deletion could be tolerated without having a significant effect on the animal health status. Indeed,

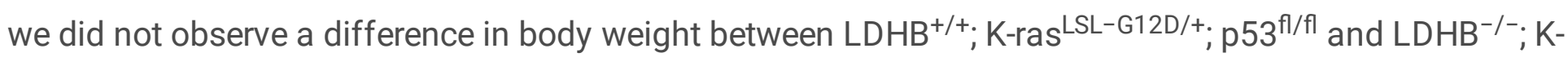
ras $^{\mathrm{LSL}-\mathrm{G} 12 \mathrm{D} /+} ; \mathrm{p53}^{\mathrm{fl} / \mathrm{fl}}$ animals (Fig. 6c). Meanwhile, tumor-initiation was detectable in $\mathrm{LDHB}^{+/+} ; \mathrm{K}-$ ras $^{\mathrm{LSL}-\mathrm{G} 12 \mathrm{D} /+} ; \mathrm{p} 53^{\mathrm{f} / \mathrm{fl}}$ mice at 14 weeks of age, 6 weeks after intratracheal instillation, whereas no tumor initiation was detectable in control animals. Two weeks later, a reduced number of tumors was also

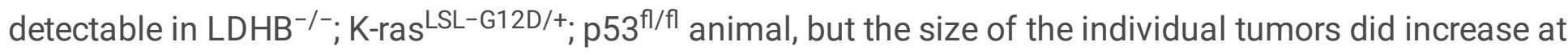
a lower rate during the whole course of the experiment (Fig. $6 \mathrm{~d}$ and supplemental Fig. S6b). These results suggest that depletion of LDHB inhibits tumorigenesis and delays tumor growth. We also observed lower disease incidence in the lungs of $\mathrm{LDHB}^{-/-} ; \mathrm{K}^{-} \mathrm{ras}^{\mathrm{LSL}-\mathrm{G} 12 \mathrm{D} /{ }^{+}} ; \mathrm{p} 53^{\mathrm{fl} / \mathrm{fl}}$ mice with a 3D reconstruction of microCT images (Fig. 6e). Moreover, at the end of the experiment, the average weight of lungs from $\mathrm{LDHB}^{-/-} ; \mathrm{K}^{-r a s}{ }^{\mathrm{LSL}-\mathrm{G} 12 \mathrm{D} /+} ; \mathrm{p} 53^{\mathrm{fl} / \mathrm{fl}}$ animals was significantly lower, and fewer tumor nodules were

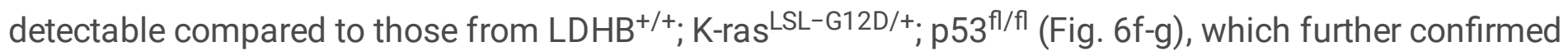
our conclusion. Moreover, our in silico analysis based on a GEO dataset also revealed that LDHB expression was increased in oncogene-induced lung dysplasia compared to normal lung tissue (Supplementary Fig. S6c), which further supports our conclusion that LDHB is important for tumorigenesis.

\section{Lactate supplementation increases survival of patient- derived tumor tissue ex vivo}


Our in silico analysis revealed that LDHB expression was higher in tumor tissue than in normal tissue in both lung adenocarcinoma (LUAD) and squamous cell carcinoma (LUSC) and increased LDHB expression in LUAD correlated with poor patient survival (Supplementary Fig. S7a-b). Thus, we extended our analysis of lactate-related metabolism to patient-derived tumor tissue cultured ex vivo (Fig. 7a-b). Indeed, supplementation of $20 \mathrm{mM}$ sodium lactate to growth medium containing $2.5 \mathrm{mM}$ or $10 \mathrm{mM}$ glucose, the physiological lactate concentration of healthy and inflamed tissues including the tumor microenvironment, respectively (48), significantly increased survival of lung tumor cells (Fig. 7c, and Supplementary Fig. S7c) further corroborating our finding that LDHB-dependent lactate metabolism is critical for human lung cancer cell survival.

\section{Discussion}

Guided by the metabolic symbiosis model (reviewed in (18), we hypothesized that, in the A549 cell line, the tumor-initiating holoclonal subpopulation is dependent on LDHB to metabolize the lactate produced by the more mesenchymal paraclonal subpopulation (29). Indeed, LDHB silencing not only diminished the fraction of holoclonal A549 cells but also decreased the fraction of GLDC-positive cells (Fig. 3b). Intriguingly, lactate preferentially rescued the survival of the holoclonal A549 subpopulation and increased the fraction of the GLDC ${ }^{+}$subpopulation (Fig. 1c-d and Supplementary Fig. S1e). In the context of maintaining a stem-like phenotype, it was shown that retinoid (vitamin A) is oxidized to Retinal, which serves as a substrate for ALDH1 to synthesize retinoic acid, e.g., a metabolite essential for differentiation during early embryonic development and the maintenance of cancer stem cell subpopulations in tumors (reviewed in (49). In agreement with the dramatically reduced aldehyde activity in all three-tested cell lines (Fig. 3c and Supplementary Fig. S3d), retinoid was one of the most abundantly accumulated metabolites upon LDHB silencing (supplementary Excel file Metabolomics Data). Further, expression of the most abundantly expressed aldehyde dehydrogenase isoforms, e.g., ALDH1A1 and ALDH3A1, which are 1.6and 7.1-fold overexpressed in the stem-like holoclonal compared to the more mesenchymal paraclonal A549 cells (29), was also significantly reduced upon LDHB silencing (supplementary Excel file Gene Expression). Thus, in agreement with reducing the $\mathrm{GLDC}^{+}$and $\mathrm{ALDH}^{+}$subpopulations, $\mathrm{LDHB}$ silencing reduced the synthesis of pyrimidine and retinoic acid, both of which are critically associated with maintaining a stem-like state linked with increased tumor initiation capacity. However, additional studies will be necessary to elucidate on the molecular level how the modulation of LDHB expression affects the complex interplay between lactate metabolism and stemness pathways that are associated with an increased tumor initiation capacity.

It has been shown that silencing of LDHA decreases glycolysis and leads to a reactivation of mitochondrial function in breast, lung, liver, lymphoma, and pancreatic cancers (reviewed in (50). Thus, we speculated that silencing LDHB should result in lactate accumulation and blunting of mitochondrial metabolism. Indeed, LDHB silencing decreased OCR (Fig. 2e). Surprisingly, intracellular lactate levels were also significantly decreased upon LDHB silencing (supplementary Excel file Metabolomics Data), as was 
lactate-dependent ECAR (Supplementary Fig. 2d). Lactate is the end product of glycolysis. Thus our results suggested that LDHB silencing inhibits glycolysis. Indeed, the levels of glycolytic intermediates were significantly depleted upon LDHB silencing, as were the levels of metabolites of the PPP, which is fueled by glycolytic intermediates (Fig. $5 \mathrm{~d}$ ). In agreement, our Seahorse analysis indicated that glycolysis is significantly inhibited upon LDHB silencing in all tested cell lines (Supplementary Fig. S2d). In summary, in contrast to the inhibition of LDHA, silencing of LDHB blunts both mitochondrial metabolism and glycolysis.

Interestingly, mitochondrial DNA integrity was not restored after LDHB silencing (Fig. 4c), so LDHB silencing resulted in a phenotype similar to that observed in Rho 0 cells. In detail, long-term treatment with a low dose of the DNA damage-inducing agent Ethidium Bromide results in complete depletion of mitochondrial DNA, the definition of Rho 0 cells (51). Consequently, Rho 0 cells rely on medium supplementation with uridine to compensate for the loss of the ETC complex III, which is encoded by mitochondrial DNA and is essential for the function of DHODH, e.g., pyrimidine de novo synthesis. Intriguingly, although Rho 0 cancer cells retained their proliferation ability, they completely lost their tumor initiation capacity (52), thus resembling cancer cells adapted to long-term LDHB silencing, e.g., A549 shLDHB clones (data not shown). Strikingly, the generation of tumors in syngeneic mice by Rho 0 cancer cells is linked to the acquisition of the host mtDNA from stromal cells and subsequent restoration of the OXPHOS activity (53). Indeed, we also observed that tumor initiation was dramatically reduced but not completely abrogated, both in xenografts of A549 shLDHB clones (Fig. 3g) and also in LDHB knockout animals (Fig. 6d). Thus, it will be interesting to elucidate how cellular metabolism can be restored upon LDHB silencing, e.g., either by the acquisition of mitochondria or by rewiring the cellular metabolism.

Regarding the differences in metabolic states, a shortcoming of our study is that we performed neither the gene expression analysis nor the metabolomics at the single-cell level. Indeed, single-cell RNAsequencing of cancer cells revealed significant differences in LDHB expression between and within cancer, stroma, and immune subpopulations of primary breast cancer tumors (54) (see also https://www.ebi.ac.uk/gxa/sc/experiments/E-GEOD-75688/results?geneld=ENSG00000111716). Our microscopic analysis revealed that LDHB expression in the xenografts was highly heterogeneous (Supplementary Fig. S3i), confirming that lactate-mediated metabolic symbiosis might occur not only between cancer cells but also between all cell types within a tumor. Nevertheless, lactate supplementation generally increased survival in patient-derived ex vivo tumor tissues (Fig. 7c and Supplementary Fig. 7c), consistent with the finding that lactate is the primary carbon source for the TCA cycle in vivo and provides substrate and electrons for oxidative phosphorylation $(16,17)$. It will be interesting to dissect how the underlying genetic background and the various gradients, e.g., $\mathrm{pH}$, hypoxia, metabolites, affect lactate-mediated metabolic symbiosis between single cells and the cellular subpopulations in the complex tumor microenvironment.

In the context of persistent mtDNA damage, mitochondrial DNA was recently shown to serve as a cellular genotoxic stress sentinel (38). Intriguingly, failure to restore mtDNA integrity may be an active process leading to the release of damaged mtDNA into the cytoplasm, thereby serving as a second messenger 
signaling cellular stress (38). Specifically, once released into the cytoplasm, mtDNA activates not only the cyclic GMP-AMP synthase (cGAS)-STING (stimulator of interferon genes) dependent innate immune signaling pathway but also activates Toll-like receptor 9, inflammasomes (most notably Nlrp3), and other nucleic acid sensors in the cytoplasm (38). Consistently, we found that most of the signaling pathways whose gene expression was increased after LDHB silencing were related to immune activation (Supplementary Fig. S4b). However, the mechanisms underlying the release of mtDNA into the cytoplasm have not yet been defined, nor have the numerous up- and downstream effectors that orchestrate the sentinel function of the mtDNA release. In addition, LDHB silencing affects lactate metabolism and pH regulation, both of which are intrinsically linked to the regulation of the immune response (55). Thus, our study suggests that by triggering the sentinel function of mtDNA upon its depletion, LDHB may serve as an integral component of an evolutionarily conserved and actively maintained pathway to trigger immune activation in the presence of aberrant lactate metabolism.

In summary, our results lead us to propose a working model: consistent with the "reverse Warburg effect" model, a mesenchymal subpopulation of NSCLC cells exhibits increased glucose uptake, which is converted to lactate via LDHA-dependent glycolysis. The secreted lactate fuels TICs featuring cancer stem cell markers, e.g., increased GLDC expression, OXPHOS, and ALDH activity. TICs convert lactate in an LDHB-dependent manner to pyruvate, which subsequently drives mitochondria-dependent metabolism, including nucleotide synthesis required for nuclear and mtDNA replication and maintenance, as well as $\mathrm{NAD}^{+} / \mathrm{NADH}$ synthesis. Mitochondrial DNA integrity cannot be restored, which is associated with a continuously reduced mitochondrial complex activity and OXPHOS.

Interestingly, the activity of individual mitochondrial complexes encoded by mitochondrial DNA remained reduced regardless of substrate availability during long-term adaptation (Fig. 4e and Supplementary Fig. S4d), suggesting that it is indeed the OXPHOS capacity per se that is reduced, rather than the supply of OXPHOS substrates, i.e., the energetic products of the TCA cycle. Decreased OXPHOS activity further decreases pyrimidine synthesis, reducing stemness and thus sphere and tumor initiation ability. Furthermore, our results revealed that LDHB silencing triggers an innate immune response. We speculate that LDHB silencing leads to deregulation of mtDNA replication, which may elicit the release of mtDNA into the cytosol, thereby triggering a STING-dependent innate immune response (56), as recently shown in response to cellular pyrimidine imbalance (57).

In summary, our work contributes to the growing body of evidence suggesting that lactate metabolism is an important determinant of cancer stem cell maintenance and that nucleotide metabolism at the molecular level is inextricably linked to an increased tumor initiating capacity and tumorigenesis. Surprisingly, our study also revealed a link between LDHB-mediated lactate metabolism and the persistent accumulation of DNA damage of mitochondrial DNA, which is not only associated with a reduced capacity for tumorigenesis, mimicking Rho 0 cells but also with the activation of innate immune response. Thus, our study may provide the basis for the development of future therapies based on targeting LDHB in combination with agents that affect cellular plasticity, induction of DNA damage, or even immunotherapy. 


\section{Methods}

\section{Cell culture}

All cell lines were obtained from American Type Culture Collection (Manassas, VA, USA), except patientderived primary LUAD cells PF139 were established as recently reported (58). Cells were maintained in the RPMI medium (Cat. \#8758; Sigma-Aldrich) or DMEM/F12(Cat. \#21331020; Life Technologies) supplemented with 2mM L-glutamine (Cat. \#25030024, Life Technologies); $10 \%$ fetal bovine serum/FBS (Cat. \#10270-106; Life Technologies) and 1\% penicillin/streptomycin solution (Cat. \#P0781, SigmaAldrich). The cells were authenticated by DNA fingerprinting and confirmed free from mycoplasma contamination (Microsynth, Bern, Switzerland) at $37^{\circ} \mathrm{C}$ in a humidified $5 \% \mathrm{CO}_{2}$ incubator.

\section{Oxygen Consumption Rate (OCR) and Extracellular Acidification Rate (ECAR)}

Oxygen consumption rate and extracellular acidification rate were measured using the XFe96 Extracellular Flux Analyzer (Agilent Technologies). 18,000 PARA, A549, H838, and 20,000 HOLO, H358, H460, PF139 cells were seeded overnight in Seahorse XF96 V3 PS cell culture microplates (Cat. \#101085004; Agilent Technologies) and reached $80-90 \%$ confluence on the day of the experiment. The XF sensor cartridges were hydrated with sterile ddH2O in a $\mathrm{CO} 2$-free incubator at $37^{\circ} \mathrm{C}$. For Mito Stress Test, cells were washed twice and changed into Seahorse XF DMEM or RPMI medium (Cat. \#103680-100 and 103681-100, Agilent Technologies) containing $10 \mathrm{mM}$ glucose, $0.5 \mathrm{mM}$ pyruvate, $2 \mathrm{mM}$ glutamine adjusted to $\mathrm{pH}$ 7.4. Then the cells were incubated in a CO2-free incubator for $1 \mathrm{~h}$. Then, $1 \mu \mathrm{M}$ oligomycin, $1.0 \mu \mathrm{M}$ and $1.5 \mu \mathrm{M}$ FCCP, a mixture of $1 \mu \mathrm{M}$ rotenone and $1 \mu \mathrm{M}$ antimycin A were added successively. For the Glycolysis Stress Test, cells were washed twice and changed into Seahorse XF DMEM, or RPMI medium spiked with $2 \mathrm{mM}$ glutamine and adjusted to $\mathrm{pH}$ 7.4. The cells were then incubated in a CO2-free incubator for $1 \mathrm{~h}$. Then $10 \mathrm{mM}$ glucose, $1 \mu \mathrm{M}$ oligomycin, $50 \mathrm{mM}$ 2-DG were added successively. All chemicals are listed in Table T2. OCR, ECAR was measured using the Seahorse XFe96 analyzer (Agilent Technologies). The data were analyzed with Seahorse Wave (Agilent Technologies). All raw data is normalized to 50 ng DNA, which is quantified by CyQUANT ${ }^{\mathrm{TM}}$ Cell Proliferation Assay kit (Cat. \# C7026; Thermo Fisher Scientific) according to the manufacturer's protocol. Respiration parameters were determined as follows: Basal respiration as basal OCR; ATP-linked respiration by subtracting proton leak from basal OCR; spare respiration as the difference between maximal respiration and basal respiration; maximal OCR calculated as the difference of antimycin plus rotenone rate from FCCP rate. Basal ECAR as glycolysis; maximal glycolysis as glycolytic capacity achieved by the additional oligomycin injection; glycolytic reserve as the difference between maximal and basal ECAR.

\section{D colony formation assay and 3D sphere formation assay}


For the 2D colony formation assay, 500 cells were cultivated in 6-well plates for 7-14 days. Colonies were stained with crystal violet ( $0.5 \%$ dissolved in $25 \%$ methanol). The number of colonies was counted using Fiji (Fiji, RRID:SCR_002285) as described $(59,60)$.

For the 3D colony formation assay, 500 cells were cultured in $2 \mathrm{~mL}$ CnT-Prime Airway Epithelial Proliferation Medium (Cat. \#CnT-PR-A; CELLNTEC) supplemented with $0.5 \%$ methylcellulose (Cat. \#M0262; Sigma-Aldrich); 10ng/mL Human IGF-II (Cat. \#100-12; PeproTech); 10ng/mL Human Heregulin $\beta$-1 (Cat. \#AF-100-03; PeproTech); 1 MM DMH-1 (Cat. \#73632; STEMCELL Technologies); $1 \mu$ M A-83-01 (Cat. \#72022; STEMCELL Technologies); 1\% penicillin/streptomycin solution (Cat. \#P0781, Sigma-Aldrich). in Nunclon Sphera 6-Well Plate (Cat. \#174932; Thermo Fisher Scientific). After 2-3 weeks, spheres were counted under the microscope, and images were taken at 40X magnification.

\section{Nucleotide rescue experiment}

For the proliferation rescue experiment, cells were plated in triplicate into 6-well plates with an initial seeding density of $0.1 \times 10^{6}$ cells per well for overnight equilibration. Subsequently, cells were changed to a medium with $100 \mu \mathrm{M}$ hypoxanthine (Cat. \# H9377; Sigma-Aldrich), $100 \mu \mathrm{M}$ adenine (Cat. \#A8626, Sigma-Aldrich), $400 \mu \mathrm{M}$ uridine (Cat. \#U6391; Sigma-Aldrich), or solvents ( $\mathrm{NaOH}$ and $\mathrm{HCl}$ ). According to the established methods or solvents $(\mathrm{NaOH}$ and $\mathrm{HCl})$. According to the established procedure, cell number was quantified after 5 days using a hemacytometer (Cat. \#Z359629; Merck) (61). The proliferation rate was calculated using the following formula:

Proliferation rate (doublings per day) $=\log _{2}($ final cell count(day5)/initial cell count(day 1$\left.)\right) / 4($ days $)$

For the sphere formation rescue experiment, 500 cells were cultured in the CnT-Prime medium, as described above, with the indicated concentration of hypoxanthine, adenine, uridine, or solvents. After 2-3 weeks, the spheres were counted as described above.

\section{Flow cytometry}

Cells were harvested as indicated above. Cells were stained with antibodies against surface markers for 30 minutes on the ice for the extracellular staining, protected from light. For the intracellular staining, cells were fixed with IC fixation buffer (Cat. \#00-8222-49; Thermo Fisher Scientific) for 15 minutes and permeabilized with $0.1 \%$ Triton X100 (Cat. \#X100; Sigma-Aldrich) for 10 minutes at room temperature. Then, cells were incubated in $200 \mu \mathrm{L}$ PBS containing $2 \%$ FBS and $0.25 \%$ Fc Receptor Binding Inhibitor Functional Grade Monoclonal Antibody for 10 minutes at room temperature. Subsequently, cells were stained with intracellular markers overnight on a rotating wheel $(3 \mathrm{rpm})$ at $4^{\circ} \mathrm{C}$ and protected from light. Finally, cells were washed three times with $2 \%$ FBS and resuspended in $2 \%$ FBS containing $0.5 \mathrm{~g} / \mathrm{mL}$ DAPI. All samples were measured on a BD Bioscience LSR2 upgraded flow cytometer, and 10,000 events were recorded. FlowJo V10 (Tree Star, Inc. (Ashland, OR, USA, FlowJo, RRID: SCR_008520)) was used to 
analyze FCS files. All antibodies are list in Table T1. For the ALDEFLUOR (Cat. \#01700; STEMCELL Technologies) and Reactive oxygen species (ROS) (Cat. \#K936-250; BioVision), staining was performed according to the manufacturer's protocol (https://cdn.stemcell.com/media/files/pis/29888-PIS_1_1_2.pdf and https://www.biovision.com/documentation/datasheets/K936.pdf), then measured and analyzed as above.

\section{Cell viability assay}

Scramble control and siLDHB cells were plated at 3000 cells/well in 96-well plates for overnight culture. For the lactate rescue experiment, cells were changed into DMEM no glucose medium (cat \#11966025; Thermo Scientific) containing 20mM L-lactic acid (cat \#27714; Sigma-Aldrich) or HCl and adjusted to $\mathrm{pH}$ 6.8. For the nucleotide rescue experiment, different concentrations of hypoxanthine, adenine, and uridine or solvents were added to the cells in DMEM/F12 medium. For the drug sensitivity assay, cells were treated with different concentrations of rotenone (cat. \#ab143145; Abcam), UK5099 (cat. \#PZ0160; Sigma-Aldrich), 2-deoxy-D-glucose (cat. \#D8375; Sigma-Aldrich), 5'-deoxy-5-fluorocytidine (cat. 66335-384; Cayman Chemical Company), teriflunomide (cat. \#S4169; Selleck Chemicals) in DMEM/F12 medium. After 4 days, cell viability was determined by Acid Phosphatase (APH) Assay according to the protocol described previously (62). Absorbance was quantified in a Tecan Infinite ${ }^{\circledR}$ M1000 Microplate Reader.

\section{Immunoblotting}

Cell lysates were extracted in 1X RIPA Lysis and Extraction Buffer (Cat. \#89901; Thermo Fisher Scientific) with 2X Protease and Phosphatase Inhibitor Cocktail (Cat. \#78440; Thermo Fisher Scientific) for 20 minutes on ice. The lysate was purified by centrifugation at $14,000 \mathrm{~g}$ for 25 minutes at $4^{\circ} \mathrm{C}$. Protein concentration was quantified using the BCA Protein Assay Kit (Cat. \#23209; Thermo Fisher Scientific). Samples were resolved by SDS-PAGE and then transferred using Trans-Blot ${ }^{\circledR}$ Turbo ${ }^{\text {TM }}$ Mini Nitrocellulose Transfer Packs (Cat. \#1704158; Bio-Rad). Prior to staining with antibodies, membranes were blocked with TBS (Cat. \#927-60001; LI-COR Biosciences) for 1 hour at room temperature. Subsequently, the membranes were incubated with the primary antibodies overnight on a rotating wheel $(3 \mathrm{rpm})$ at $4^{\circ} \mathrm{C}$. After washing three times with TBS wash buffer (Tris Buffered Saline (1 tablet/500mL; Cat. \#9415810TAB; Sigma Aldrich Chemie GmbH) + 2\% TWEEN 20(Cat. \#P1379; Sigma-Aldrich)), the membranes were incubated with secondary antibodies of IRDye 680LT-conjugated goat anti-mouse lgG (1: 5000; (LICORBiosciences Cat\# 926-68020, RRID:AB_10706161), and IRDye 800CW-conjugated goat anti-rabbit IgG (1:5,000; LI-COR Biosciences Cat\# 926-32211, RRID:AB_621843) were incubated for 30 minutes at room temperature. All primary antibodies are listed in Table T1. Images were acquired and analyzed using the Odyssey Infrared Imaging System (Li-COR Biosciences).

\section{Immunofluorescence microscopy}


Cells grew on 4-well chamber slides (cat. \#154526; Thermo Scientific Nunc) and reached 50-80\% confluency. Cells were then fixed with $4 \%$ paraformaldehyde for 20 min at RT and permeabilized with $0.2 \%$ Triton X-100 for 15 min. The cells were then treated with acetone and methanol (1:1) for 20 minutes at room temperature. After the cells were blocked with 1\% BSA for 2 hours at room temperature, the cells were stained with the antibodies listed in Table T1. Then cells were mounted mount buffer containing DAPI (Cat. \# P-3693; Thermo Fisher Scientific). For mitochondrial mass staining, cells were pretreated with 200nM MitoTracker ${ }^{\circledR}$ Deep Red FM (Cat. \#M22426; Thermo Fisher Scientific) for 1 hour in a cell culture incubator. Then procedures were continued as described above. Images were acquired by ZEISS_LSM 710 confocal microscope and processed by Fiji.

\section{Immunohistochemistry}

Immunohistochemistry was performed at room temperature using the fully automated BOND RX® staining system (Leica Biosystems) as previously described (63). Samples were stained with appropriate antibodies (Table T1). Images were acquired and processed using Fiji.

\section{LDHB activity}

LDHB activity was measured by the LDH-B Activity Assay kit (Cat. \#ab140361; Abcam) according to the manufacturer's instructions.

\section{RNA Sequencing and data analysis}

After gene silencing by siRNA, RNA sequencing was performed from A549 (NCI-DTP Cat\# A549, RRID:CVCL_0023) and H358 (NCl-DTP Cat\# NCl-H358, RRID:CVCL_1559) cell cultures. Total RNA was isolated and purified with RNeasy Mini Kit (Cat. \#74106, Qiagen). The quantity and quality of the purified total RNA were assessed using a Thermo Fisher Scientific Qubit 4.0 fluorometer with the Qubit RNA BR Assay Kit (Cat. \#Q10211; Thermo Fisher Scientific) and an Advanced Analytical Fragment Analyzer System using a Fragment Analyzer RNA Kit (Cat. \#DNF-471; Agilent), respectively. Sequencing libraries were made using an illumina TruSeq Stranded mRNA Library Prep kit (Cat. \#20020595; illumina) combined with TruSeq RNA UD Indexes (Cat. \#20022371; illumina) according to illumina's guidelines. Pooled cDNA libraries were sequenced paired-end using an illumina NovaSeq 6000 SP Reagent Kit (Cat. \#20028401, 100 cycles; illumina) on an illumina NovaSeq 6000 instrument. The run produced, on average, 41 million reads/sample. The quality of the sequencing run was assessed using illumina Sequencing Analysis Viewer (illumina version 2.4.7) and all base call files were demultiplexed and converted into FASTQ files using illumina bcl2fastq conversion software v2.20. The quality control assessments, generation of libraries, and sequencing were conducted by the Next Generation Sequencing Platform, University of Bern. 
Pathway enrichment analysis was performed using Metascape (RRID:SCR_016620), and the Gene Set Enrichment Analysis (GSEA) was performed in GSEA software (SeqGSEA, RRID:SCR_005724) (64-66). The gene set of Mitochondrial respiratory chain complexes was acquired from HUGO Gene Nomenclature Committee and analyzed with GSEA using R 4.0.2.

\section{Gene silencing by small interfering (siRNA) and short hairpin RNAs (shRNA)}

For transient knockdown, cells were cultured in 6-well plates overnight until 50-70\% confluence was achieved. Cells were then transfected with Lipofectamine 2000 (Cat. \#11668027; Invitrogen) and pooled LDHB human siRNA Oligo Duplex (Cat. \#SR320835; Origene) according to the manufacturer's protocol. The Trilencer-27 Universal Scrambled Negative Control siRNA Duplex was used as a negative control.

For stable knockdown, the LDHB Human shRNA Plasmid Kit (Cat. \#TL311768; Origene) was used to produce lentivirus according to The RNAi Consortium (TRC) Broad Institute protocol. The shRNA sequences are listed as follows: Control shRNA: Sense Target strand GCACTACCAGAGCTAACTCAGATAGTACT, Loop TCAAGAG, Antisense Target strand AGTACTATCTGAGTTAGCTCTGGTAGTGC; shLDHB-1 Sense Target strand TGAATGTGGCAGGTGTTTCTCTCCAGGAA, Loop TCAAGAG, Antisense Target strand TTCCTGGAGAGAAACACCTGCCACATTCA; shLDHB-2 Sense Target strand AGTCTCTGGCTGATGAACTTGCTCTTGTG, Loop TCAAGAG, Antisense Target strand CACAAGAGCAAGTTCATCAGCCAGAGACT. Cells were infected with lentivirus to generate stable LDHB knockdown or scramble control cell lines according to the protocol from Addgene (https://www.addgene.org/protocols/generating-stable-cell-lines/). After infection, cells were selected after 3 days of treatment with $2 \mu \mathrm{g} / \mathrm{mL}$ puromycin. The cells selected with puromycin were then sorted using the GFP marker by flow cytometer. Subsequently, 100 cells were plated in $150 \mathrm{mmX} 20 \mathrm{~mm}$ cell culture dishes (cat \#20151; Bioswisstec) treated with $2 \mu \mathrm{g} / \mathrm{mL}$ puromycin. Isolated colonies were identified microscopically. The medium was aspirated and washed with PBS. 8-mm cloning cylinders (cat. \#TR-1004; MERCK Millipore) were placed around individual clones and filled with $200 \mu \mathrm{l}$ of $1 \mathrm{x}$ TrypLE (cat. \#A1217702; Thermo Fisher Scientific). After incubation at $37^{\circ} \mathrm{C}$ for $5 \mathrm{~min}$, detached cells were transferred to tissue culture-treated 6-well plates (Cat. \#174932; Thermo Fisher Scientific) and cultured to $80-90 \%$ confluence for 6 days. Individual clones were verified by Western blot.

\section{Mitochondrial complex activity}

Two million cells were harvested and resuspended in a mitochondrial respiration medium prepared according to the protocol from Oroboros (https://www.bioblast.at/images/d/d9/MiPNet14.13_MediumMiR06.pdf). Mitochondrial complex I, II, IV activity is quantified using Oroboros 02k (Bioblast, Austria) 
according to the previously described protocol (67). All chemicals are listed in Table T2. The data were acquired and processed with DatLab4.0 (Bioblat).

Quantitative real-time PCR (qRT-PCR)

Total cell DNA was extracted and purified using GenElute ${ }^{\mathrm{TM}}$ Mammalian Genomic DNA Miniprep Kit (Cat. \#G1N350; Sigma). DNA concentration was quantified by Nanodrop 2000 (Thermo Fisher Scientific). A protocol published previously (68) has been adapted to measure DNA damage by real-time PCR (rtPCR). The method is based on using a DNA polymerase capable of generating long DNA fragments (LRPCR), a process blocked by DNA damage. Thus, target genomic DNA containing lesions will be less amplified compared to a non-damaged control genomic DNA. In this method, 3 different gene fragments are targeted: $\beta$-globin (13kb, 5' region, non-transcribed), Hprt (10.4kb, transcribed), and Mito (8.9kb, lack of histones). All primers were synthesized and ordered from Microsynth, see Table T3. The qRT-PCR was performed in 96-well plates. For SRPCR, $10 \mu \mathrm{L}$ of reaction mix for each well contained $15 \mathrm{ng}$ of DNA, $1 \mu \mathrm{M}$ $\beta$-globin primer or $1 \mu \mathrm{M}$ Mito DNA primer and $5 \mu \mathrm{L}$ GoTaq@ qPCR master mix (2x) (Cat. \#A6002; Promega), $2 \mu \mathrm{L}$ nuclease-free water. Run method was as follows: Hold stage $95.0^{\circ} \mathrm{C} 2$ minutes; cycling stage Step $195.0^{\circ} \mathrm{C}, 3$ seconds, Step $268.0^{\circ} \mathrm{C} 30$ seconds, 40 cycles; melt curve stage using a continuous method Step $195.0^{\circ} \mathrm{C} 15$ seconds, Step2 50.0 $0^{\circ} \mathrm{C} 1$ minute, Step3 $95.0^{\circ} \mathrm{C} 15$ seconds, Step $60.0^{\circ} \mathrm{C} 15$ seconds. For LRPCR, $20 \mu \mathrm{L}$ of reaction mix for each well contained $30 \mathrm{ng}$ of DNA, $0.2 \mu \mathrm{M} \beta$-globin primer or $1 \mu \mathrm{M}$ Mito DNA primer or $1 \mu \mathrm{M}$ Hprt primer, and $10 \mu \mathrm{L}$ GoTaq@ Long qPCR Master Mix (Cat. \#M4021; Promega), $2 \mu \mathrm{L}$ 1:4 diluted EvaGreen ${ }^{\circledR}$ Dye 20X in water (cat. \#31000; Biotium), 0. $2 \mu \mathrm{L}$ CXR reference dye (cat. \#C5411; Promega), $1.8 \mu \mathrm{L}$ nuclease-free water. Run method was as follows: Hold stage $95.0^{\circ} \mathrm{C} 2$ minutes; cycling stage Step $192.0^{\circ} \mathrm{C}, 30$ seconds, Step2 $68.0^{\circ} \mathrm{C} 15$ minutes, 40 cycles; melt curve stage using a continuous method Step $195.0^{\circ} \mathrm{C} 15$ seconds, Step2 $50.0^{\circ} \mathrm{C} 15$ seconds, Step3 $95.0^{\circ} \mathrm{C} 15$ seconds, Step $460.0^{\circ} \mathrm{C} 15$ seconds. The reactions were performed in a 7500 Fast Real-Time PCR System (Thermo Fisher Scientific), and samples were measured in triplicates. Using the $\triangle 2 \mathrm{CT}$ method, the expression of LRPCR fragments is normalized to the expression of a small genomic DNA fragment (258bp) for $\beta$-globin and Hprt, and a small mitochondrial DNA fragment (221bp) for Mito using regular short-range PCR (SRPCR).

NMR

NMR experiments were performed on a $500.13 \mathrm{MHz}$ Bruker Avance II spectrometer (Bruker BioSpin) equipped with a $5 \mathrm{~mm}$ ATM BBFO probe with a z-gradient. 13C NMR spectra were acquired using a 1D sequence with inverse gated decoupling and a flip angle of $30^{\circ}$ ("zgig30" from the Bruker pulse program library). Each 1D 13C NMR spectrum was measured using the same parameters: a nominal temperature of $275 \mathrm{~K}$, a spectral width of $34000 \mathrm{~Hz}$, a data size of $32 \mathrm{~K}$ points, 1280 transients, an acquisition time of $0.48 \mathrm{~s}$, and a relaxation delay of $4 \mathrm{~s}$. The total experiment time for the 13C NMR acquisition was about 96 min. The spectral processing was performed using the Bruker Topspin software (version 3.2, patch level 5). The free induction decays (FIDs) were exponentially weighted with a line broadening factor of $10 \mathrm{~Hz}$, Fourier-transformed, manually phased, and baseline corrected. For each sample, the $13 \mathrm{C}$ lactate peak of 
interest was integrated. The absolute integral was then converted into an $\mathrm{mM}$ value using the integral of the starting condition with a known concentration of $20 \mathrm{mM}$ lactate. For the analysis, lactate consumption values, normalized for the cell number, were calculated via subtraction of the lactate concentration value of a specific sample from $20 \mathrm{mM}$ lactate at the starting condition.

\section{LC-MS}

$5 \times 10^{5}$ scramble control cells and siLDHB cells were plated out in 6-well plates in DEME/F12 medium. After 48 hours, the medium was aspirated and then carefully washed with $2 \mathrm{~mL}$ of prewarmed $\left(37^{\circ} \mathrm{C}\right)$ wash solvent ( $75 \mathrm{mM}$ ammonium carbonate, $\mathrm{pH}$ was adjusted to 7.4 with acetic acid). The wash solvent was completely aspirated. $400 \mu \mathrm{L}$ of the pre-cooled $\left(-20^{\circ} \mathrm{C}\right)$ extraction solvent $(40 \%$ acetonitrile, $40 \%$ methanol, $20 \%$ nanopure water) was immediately added to the plates. All chemicals are listed in Table T2. Plates were sealed with aluminum adhesive foil and immediately placed at $-20^{\circ} \mathrm{C}$. After one hour, plates were transferred to the freezer at $-80^{\circ} \mathrm{C}$. The metabolites were extracted by scraping the surface of the plates with a cell scraper on ice. Transfer the extract to a $2 \mathrm{~mL}$ Eppendorf tube and spin the tube at $5000 \mathrm{~g}$ for $5 \mathrm{~min}$ at $4^{\circ} \mathrm{C}$. The clean supernatants were transferred into $2 \mathrm{~mL}$ Eppendorf tubes and stored immediately at $-80^{\circ} \mathrm{C}$ for further analysis by LC-MS measurements. LC-MS measurement and analysis were described previously (69).

In vivo limiting dilution assay

The mouse experiments were performed in accordance with the animal welfare guidelines and protocols approved by the Institutional Animal Care and Ethical Committee; license number BE 8/16. Cells were cultured in vitro for 6 days before transplantation. Various tumor cell suspensions were inoculated subcutaneously (left and right flank) in $100 \mu \mathrm{L}$ serum-free medium and growth factor-reduced Matrigel (1:1) (Cat. \#356231, Corning) at limiting dilutions $(10,000,1,000,1,00,10)$. Age- and sex-matched NODscid IL2Rynull (NSG) mice maintained under specific pathogen-free conditions were used as recipients. Tumors were explanted after 6 weeks. Extreme limiting dilution analysis (ELDA) was performed as described (70).

Genetically engineered mouse model

The LDHB deficient sperms were ordered from the International Mouse Strain Resource (IMSR) platform, and oocytes were obtained from superovulation mature female KP mice, which were used to perform in vitro fertilization (IVF). Subsequently, the embryos were transferred to recipient females. The genotype of

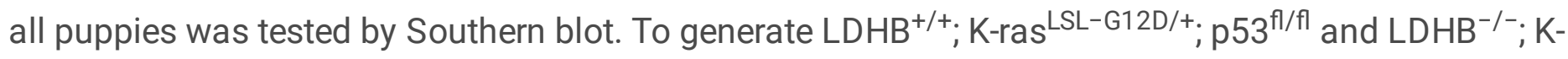
ras $^{\mathrm{LSL}-\mathrm{G} 12 \mathrm{D} /+} ; \mathrm{p} 53^{\mathrm{fl} / \mathrm{fl}}, 2.5 \times 10^{7} \mathrm{PFU}$ AAV-Cre virus in a total volume of $50 \mu \mathrm{L}$ was introduced to 8-week-old

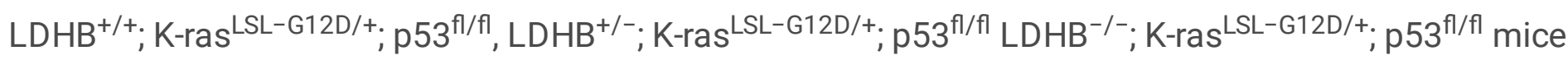
after anesthesia. Two weeks later, all mice were scanned with microCT (X-RAD SmART - Precision X-Ray) to determine the basal line for the lung. Subsequently, mice were scanned with microCT every two weeks to assess tumor development and sacrificed at 16-18 weeks of age. The microCT images were processed with Fiji and 3D Slicer version 4.13. 
The experiments with human patient samples were performed in accordance with the Declaration of Helsinki and protocols approved by the local ethics committee of the Canton of Bern; license number PB_2016-01560. In detail, NSCLC tissue specimens were acquired in collaboration with the Tissue Bank Bern. Patients of both sexes were included; age and weight were not matched. A solution of $4 \%(w / w)$ low gelling agarose (Cat. \#A9414-100G; Sigma-Aldrich) in DMEM cell culture medium was prepared and heated in a microwave $(700 \mathrm{~W})$ for a few seconds until obtaining a homogenous mix. The solution was then cooled down and kept at $37-38^{\circ} \mathrm{C}$, ready to use. Meanwhile, the NSLC tumors resected from patients were manually cleaned from any surrounding residual lung parenchyma and subsequently cut into regular cubes with an edge length of approximately $1 \mathrm{~cm}$. The cubes were embedded in the low gelling agarose solution that solidified on ice for about $15 / 20 \mathrm{~min}$. The agarose-embedded piece of the tumor is transferred to a cutting apparatus, which was in-house designed and made, resulting in PCTS of $500 \mu \mathrm{m}$ thickness. The slices, of irregular shapes, were homogenized using a round tissue punch of $6 \mathrm{~mm}$ diameter. The slices were plated in 6-well plates (one slice per well), and $1750 \mu \mathrm{l}$ of medium were added per well. PCTS were cultured at $37^{\circ} \mathrm{C}$ with $5 \% \mathrm{CO}_{2}$. Viability of the slices with complete Medium (DMEM 4.5g/L D-Glucose, Cat. \#41966-052; Gibco) was assessed up to 4 days post-incubation. Slices (in duplicates) were cultured with four different medium conditions: condition 1, $2.5 \mathrm{mM}$ glucose DMEM Medium with no lactate; condition 2, $2.5 \mathrm{mM}$ glucose DMEM medium with $20 \mathrm{mM}$ lactate; condition 3, 10 $\mathrm{mM}$ glucose DMEM medium without lactate; condition 4, $2.5 \mathrm{mM}$ glucose DMEM Medium with $20 \mathrm{mM}$ lactate.

In vivo imaging of PCTS was performed 3 days post-incubation at a confocal microscope Zeiss LSM 880. Slices were removed from the cell culture medium, quickly washed twice in Ringer's solution, and stained for 30 minutes with the kit LIVE/DEAD ${ }^{\text {TM }}$ Viability/Cytotoxicity Kit, for mammalian cells (Cat. \#L3224; Thermofisher) and HOECHST suspended in a solution of HEPES 0.01 M (Cat. \#15630-049; Gibco) and Vitamin C 140 mM in Ringer's. At the end of the incubation, slices were washed twice and mounted on a MatTek glass-bottom microwell dish ( $35 \mathrm{~mm}$ No. 0 ) for live imaging. The whole slices were imaged in tile scan mode, and stitching processing was then applied. Maximum Intensity Projection of the acquired z-stacks was obtained and finally used for image analysis and image visualization. The complete experiment was repeated twice, and the analysis was randomized.

All images were analyzed using Fiji with the same brightness, contrast, and threshold settings for each channel and experiment. Calcein AM staining (green), EthD-1 staining (red), and HOECHST staining (blue) signals were area determined and restricted to the tissue sample corresponding to the TL (bright field) channel.

Public data source and analysis

Analysis of LDHB gene expression at different stages of lung cancer development in the mouse model was obtained from the GSE13963 dataset https://www.ncbi.nlm.nih.gov/geo/query/acc.cgi? 
acc $=$ GSE13963. Data for correlation analysis of LDHB and stem cell markers were acquired and analyzed using cBioPortal (http://www.cbioportal.org/index.do). The single-cell sequencing data was collected and analyzed in CancerSEA (home (hrbmu.edu.cn)). Overall survival data and analysis were obtained and analyzed using the Kaplan-Meier plotter (Kaplan-Meier plotter [Lung] (kmplot.com)).

\section{Statistical analysis}

Statistical analysis was performed using GraphPad Prism 9 (GraphPad Prism, RRID: SCR_002798). Repeated measurements were made on different biological samples. Pearson R was reported for correlation analysis. The statistical significance of the correlation was determined by a two-tailed t-test. Error bars represent mean \pm standard deviation (SD) or mean \pm standard error of the mean (SEM). Oneway ANOVA and two-way ANOVA were followed with Tukey's multiple comparison test. Two-tailed unpaired or paired Student's t-tests were performed, as described in figure legends. The p-values $<0.05$ were considered significant. In all analyses, the significance level is presented as follows: ${ }^{*} P 0.05, \star \star P<$ $0.01, * \star \star P<0.001, * \star \star \star P<0.0001 *$.

\section{Abbreviations}

lactate dehydrogenase (LDH); lactate dehydrogenase A (LDHA); lactate dehydrogenase B (LDHB); nonsmall-cell lung cancers (NSCLC); tumor-initiating cells (TICs); glycine decarboxylase (GLDC); extreme limiting dilution analysis (ELDA); hexokinase 2 (HK2); Aldehyde dehydrogenase (ALDH); mitochondrial DNA (mtDNA); electron transport chain (ETC); gene set enrichment analysis (GSEA); reactive oxygen species (ROS); Oxygen consumption rate (OCR); extracellular acidification rate (ECAR); holoclonal A549 cells (HOLO); paraclonal A549 cells (PARA).

\section{Declarations}

\section{Ethics approval and consent to participate}

Mouse studies were conducted in accordance with Institutional Animal Care and Ethical Committeeapproved animal guidelines and protocols.

\section{Consent for publication}

Not applicable.

\section{Availability of data and material}


All data generated or analyzed during this study are included in this published article and its supplementary information files.

\section{Competing interests}

The authors declare that they have no competing interests.

\section{Funding}

This work was supported by the Swiss Cancer Research to TMM. The research contribution of RWP was funded by the Swiss Cancer Research and the Swiss National Science Foundation. The funding bodies were not involved in the design of the study and collection, analysis, and interpretation of data and in writing the manuscript.

\section{Authors' contributions}

Conception \& design - HD, RAS, TMM; Data acquisition - HD, YG, VT, DH, DK, TL, CU, HG, NZ, GJK, PD, TMM; Data interpretation \& analysis - HD, YG, VT, DH, DK, TL, CU, HG, GAG, RB, VD, JMN, PV, NZ, CR, AO, GJK, PD, RWP, RAS, TMM; Drafting of Manuscript - HD, YG, VT, DH TL, CU, GAG, CR, PD, RWP, RAS, GJK, TMM; Accountability for all aspects of work - HD, GJK, TMM. All authors read and approved the final manuscript.

\section{Acknowledgments}

We thank Dr. José Galván (Institute of Pathology, University of Bern) and Pamela Nicholson (Institute of Genetics, University of Bern) for technical assistance. Flow cytometry experiments were performed with the support of the FACS Lab at the University of Bern, Switzerland. Microscopy acquisition and analysis were performed with the support of the Live Cell Imaging Core Facility of the Department of Clinical Research coordinated by the Microscopy Imaging Center at the University of Bern, Switzerland. NSCLC tissue specimens were acquired in collaboration with the Tissue Bank Bern, Switzerland.

\section{References}

1. Al-Hajj M, Wicha MS, Benito-Hernandez A, Morrison SJ, Clarke MF (2003) Prospective identification of tumorigenic breast cancer cells. Proceedings of the National Academy of Sciences 100, 39833988

2. Hemmati HD et al (2003) Cancerous stem cells can arise from pediatric brain tumors. Proceedings of the National Academy of Sciences 100, 15178-15183 
3. Fang D et al (2005) A Tumorigenic Subpopulation with Stem Cell Properties in Melanomas. Cancer Res 65:9328-9337

4. Kim CFB et al (2005) Identification of Bronchioalveolar Stem Cells in Normal Lung and Lung Cancer. Cell 121:823-835

5. Hermann PC et al (2007) Distinct Populations of Cancer Stem Cells Determine Tumor Growth and Metastatic Activity in Human Pancreatic Cancer. Cell Stem Cell 1:313-323

6. Ricci-Vitiani $L$ et al (2007) Identification and expansion of human colon-cancer-initiating cells. Nature 445:111-115

7. Kreso A, Dick JE (2014) Evolution of the cancer stem cell model. Cell Stem Cell 14:275-291

8. Kreso A et al (2013) Variable clonal repopulation dynamics influence chemotherapy response in colorectal cancer. Science 339:543-548

9. Nguyen LV, Vanner R, Dirks P, Eaves CJ (2012) Cancer stem cells: an evolving concept. Nat Rev Cancer 12:133-143

10. Reya T, Morrison SJ, Clarke MF, Weissman IL (2001) Stem cells, cancer, and cancer stem cells. Nature 414:105-111

11. Zhang WC et al (2012) Glycine decarboxylase activity drives non-small cell lung cancer tumorinitiating cells and tumorigenesis. Cell 148:259-272

12. Berezowska S et al (2017) Glycine decarboxylase and HIF-1alpha expression are negative prognostic factors in primary resected early-stage non-small cell lung cancer. Virchows Arch 470:323-330

13. Thul PJ et al (2017) A subcellular map of the human proteome.Science356

14. Ye XQ et al (2011) Mitochondrial and energy metabolism-related properties as novel indicators of lung cancer stem cells. Int J Cancer 129:820-831

15. Sonveaux $P$ et al (2008) Targeting lactate-fueled respiration selectively kills hypoxic tumor cells in mice. J Clin Investig 118:3930-3942

16. Hui $S$ et al (2017) Glucose feeds the TCA cycle via circulating lactate. Nature 551:115-118

17. Faubert B et al (2017) Lactate Metabolism in Human Lung Tumors. Cell 171:358-371e359

18. Doherty JR, Cleveland JL (2013) Targeting lactate metabolism for cancer therapeutics. J Clin Invest 123:3685-3692

19. Zdralevic $\mathrm{M}$ et al (2018) Double genetic disruption of lactate dehydrogenases $A$ and $B$ is required to ablate the "Warburg effect" restricting tumor growth to oxidative metabolism. J Biol Chem 293:15947-15961

20. Feng $Y$ et al (2018) Lactate dehydrogenase A: A key player in carcinogenesis and potential target in cancer therapy. Cancer Med 7:6124-6136

21. Tang Z, Xu Z, Zhu X, Zhang J (2021) New insights into molecules and pathways of cancer metabolism and therapeutic implications. Cancer Commun (Lond) 41:16-36

22. Xie H et al (2014) Targeting Lactate Dehydrogenase-A Inhibits Tumorigenesis and Tumor Progression in Mouse Models of Lung Cancer and Impacts Tumor-Initiating Cells. Cell Metabol 
19:795-809

23. Chen YJ et al (2016) Lactate metabolism is associated with mammalian mitochondria. Nat Chem Biol 12:937-943

24. Rosso M et al (2019) Characterization of the molecular changes associated with the overexpression of a novel epithelial cadherin splice variant mRNA in a breast cancer model using proteomics and bioinformatics approaches: identification of changes in cell metabolism and an increased expression of lactate dehydrogenase B. Cancer \& metabolism 7:5

25. McCleland ML et al (2012) An integrated genomic screen identifies LDHB as an essential gene for triple-negative breast cancer. Cancer Res 72:5812-5823

26. Li C et al (2016) LDHB may be a significant predictor of poor prognosis in osteosarcoma. American journal of translational research 8:4831-4843

27. McCleland ML et al (2013) Lactate dehydrogenase $B$ is required for the growth of KRAS-dependent lung adenocarcinomas. Clinical cancer research: an official journal of the American Association for Cancer Research 19:773-784

28. Zha X et al (2011) Lactate dehydrogenase B is critical for hyperactive mTOR-mediated tumorigenesis. Cancer Res 71:13-18

29. Tieche CC et al (2019) Tumor Initiation Capacity and Therapy Resistance Are Differential Features of EMT-Related Subpopulations in the NSCLC Cell Line A549. Neoplasia 21, 185-196

30. Vassalli G (2019) Aldehyde Dehydrogenases: Not Just Markers, but Functional Regulators of Stem Cells. Stem Cells Int 3904645 (2019)

31. Faubert B et al (2017) Lactate Metabolism in Human Lung Tumors. Cell 171:358-371e359

32. Boudreau A et al (2016) Metabolic plasticity underpins innate and acquired resistance to LDHA inhibition. Nat Chem Biol 12:779-786

33. Bajzikova M et al (2019) Reactivation of Dihydroorotate Dehydrogenase-Driven Pyrimidine Biosynthesis Restores Tumor Growth of Respiration-Deficient Cancer Cells. Cell Metabol 29:399$416 \mathrm{e} 310$

34. Morrison BJ, Steel JC, Morris JC (2012) Sphere culture of murine lung cancer cell lines are enriched with cancer initiating cells. PLoS ONE 7:e49752

35. Yan B et al (2015) Mitochondrially targeted vitamin E succinate efficiently kills breast tumourinitiating cells in a complex II-dependent manner. BMC Cancer 15:401

36. Brisson L et al (2016) Lactate Dehydrogenase B Controls Lysosome Activity and Autophagy in Cancer. Cancer Cell 30:418-431

37. Karakaidos P, Rampias T (2020) Mitonuclear Interactions in the Maintenance of Mitochondrial Integrity.Life10

38. Wu Z, Sainz AG, Shadel GS (2021) Mitochondrial DNA: cellular genotoxic stress sentinel. Trends Biochem Sci. 10.1016/j.tibs.2021.05.004 
39. Wiley CD et al (2016) Mitochondrial Dysfunction Induces Senescence with a Distinct Secretory Phenotype. Cell Metabol 23:303-314

40. Bajzikova M et al (2019) Reactivation of Dihydroorotate Dehydrogenase-Driven Pyrimidine Biosynthesis Restores Tumor Growth of Respiration-Deficient Cancer Cells. Cell Metab 29:399$416 \mathrm{e} 310$

41. Siddiqui A, Ceppi $P$ (2020) A non-proliferative role of pyrimidine metabolism in cancer. Molecular Metabolism 35:100962

42. Wang $X$ et al (2017) Purine synthesis promotes maintenance of brain tumor initiating cells in glioma. Nat Neurosci 20:661-673

43. Nishimura T et al (2019) Cancer stem-like properties and gefitinib resistance are dependent on purine synthetic metabolism mediated by the mitochondrial enzyme MTHFD2. Oncogene 38:2464-2481

44. Khutornenko AA et al (2010) Pyrimidine biosynthesis links mitochondrial respiration to the p53 pathway. Proc Natl Acad Sci U S A 107:12828-12833

45. Qing $Y$ et al (2021) R-2-hydroxyglutarate attenuates aerobic glycolysis in leukemia by targeting the FTO/m(6)A/PFKP/LDHB axis. Mol Cell. 10.1016/j.molcel.2020.12.026

46. Kersten K, de Visser KE, van Miltenburg MH, Jonkers J (2017) Genetically engineered mouse models in oncology research and cancer medicine. EMBO Mol Med 9:137-153

47. Wakabayashi H, Tsuchiya M, Yoshino K, Kaku K, Shigei H (1996) Hereditary deficiency of lactate dehydrogenase H-subunit. Intern Med 35:550-554

48. Pucino V et al (2019) Lactate Buildup at the Site of Chronic Inflammation Promotes Disease by Inducing CD4(+) T Cell Metabolic Rewiring. Cell Metabol 30:1055-1074e1058

49. Mezquita B, Mezquita C (2019) Two Opposing Faces of Retinoic Acid: Induction of Stemness or Induction of Differentiation Depending on Cell-Type.Biomolecules 9

50. Glancy B et al (2021) Mitochondrial lactate metabolism: history and implications for exercise and disease. J Physiol 599:863-888

51. Gao Y et al (2019) Cisplatin-resistant A549 non-small cell lung cancer cells can be identified by increased mitochondrial mass and are sensitive to pemetrexed treatment. Cancer Cell Int 19:317

52. Nakhle J, Rodriguez AM, Vignais ML (2020) Multifaceted Roles of Mitochondrial Components and Metabolites in Metabolic Diseases and Cancer.International journal of molecular sciences21

53. Dong LF et al (2017) Horizontal transfer of whole mitochondria restores tumorigenic potential in mitochondrial DNA-deficient cancer cells.eLife6

54. Chung W et al (2017) Single-cell RNA-seq enables comprehensive tumour and immune cell profiling in primary breast cancer. Nat Commun 8:15081

55. Certo M, Tsai CH, Pucino V, Ho PC, Mauro C (2021) Lactate modulation of immune responses in inflammatory versus tumour microenvironments. Nat Rev Immunol 21:151-161

56. West AP, Shadel GS (2017) Mitochondrial DNA in innate immune responses and inflammatory pathology. Nat Rev Immunol 17:363-375 
57. Sprenger HG et al (2021) Cellular pyrimidine imbalance triggers mitochondrial DNA-dependent innate immunity. Nature metabolism 3:636-650

58. Stockhammer $P$ et al (2020) HDAC inhibition synergizes with ALK inhibitors to overcome resistance in a novel ALK mutated lung adenocarcinoma model. Lung Cancer 144:20-29

59. Schindelin J et al (2012) Fiji: an open-source platform for biological-image analysis. Nat Methods 9:676-682

60. Choudhry P (2016) High-Throughput Method for Automated Colony and Cell Counting by Digital Image Analysis Based on Edge Detection. PLoS ONE 11:e0148469-e0148469

61. Sullivan LB et al (2018) Aspartate is an endogenous metabolic limitation for tumour growth. Nat Cell Biol 20:782-788

62. Friedrich J, Seidel C, Ebner R, Kunz-Schughart LA (2009) Spheroid-based drug screen: considerations and practical approach. Nat Protoc 4:309-324

63. Berezowska S, Galván JA (2017) In: Pellicciari C, Biggiogera M (eds) "Immunohistochemical Detection of the Autophagy Markers LC3 and p62/SQSTM1 in Formalin-Fixed and ParaffinEmbedded Tissue" in Histochemistry of Single Molecules: Methods and Protocols. Springer New York, New York, NY, pp 189-194. 10.1007/978-1-4939-6788-9_13

64. Zhou Y et al (2019) Metascape provides a biologist-oriented resource for the analysis of systemslevel datasets. Nat Commun 10:1523

65. Subramanian A Gene set enrichment analysis: A knowledge-based approach for interpreting genomewide expression profiles. Proceedings of the National Academy of Sciences 102, 15545 et al (2005)

66. Mootha VK et al (2003) PGC-1a-responsive genes involved in oxidative phosphorylation are coordinately downregulated in human diabetes. Nat Genet 34:267-273

67. Au - Djafarzadeh S, Au SM, Jakob (2017) High-resolution Respirometry to Assess Mitochondrial Function in Permeabilized and Intact Cells. JoVE. doi:10.3791/54985. e54985

68. Edwards JG (2009) Quantification of mitochondrial DNA (mtDNA) damage and error rates by realtime QPCR. Mitochondrion 9:31-35

69. Alseekh $S$ et al (2021) Mass spectrometry-based metabolomics: a guide for annotation, quantification and best reporting practices. Nat Methods 18:747-756

70. Hu Y, Smyth GK (2009) Extreme limiting dilution analysis for comparing depleted and enriched populations in stem cell and other assays. J Immunol Methods 347:70-78

71. Di Gialleonardo V et al (2016) High-Throughput Indirect Quantitation of (13)C Enriched Metabolites Using (1)H NMR. Anal Chem 88:11147-11153

72. Dai Z, Locasale JW (2017) Understanding metabolism with flux analysis: From theory to application. Metab Eng 43:94-102

73. Ragavan M, Merritt ME (1996) Nuclear Magnetic Resonance Measurement of Metabolic Flux Using (13)C and (1)H Signals. Methods Mol Biol 29-40 (2019) 
74. Buescher JM et al (2015) A roadmap for interpreting (13)C metabolite labeling patterns from cells. Curr Opin Biotechnol 34:189-201

\section{Tables}

Due to technical limitations, tables are only available as a download in the Supplemental Files section.

\section{Figures}

\section{Figure 1}

TICs are characterized as OXPHOS phenotype and tend to lactate utilization.

a. The real-time oxygen consumption rate (OCR) and extracellular acidification rate (ECAR) of holoclonal (HOLO) and paraclonal (PARA) cells was measured in 96-well plates cultured in DMEM medium using the Seahorse Xfe96 Analyzer. For OCR, $1 \mu \mathrm{M}$ oligomycin, $1.0 \mu \mathrm{M}$ and $1.5 \mu \mathrm{M}$ FCCP, a mixture of $1 \mu \mathrm{M}$ rotenone and $1 \mu \mathrm{M}$ antimycin A were added sequentially $(\mathrm{n}=24)$. For ECAR, $10 \mathrm{mM}$ glucose, $1 \mu \mathrm{M}$ oligomycin, 50 mM 2-DG were added sequentially $(n=26)$. The error bar was represented with mean \pm SEM. b. A549, HOLO, and PARA cells were pretreated in DMEM medium (no glucose, no glutamine, no pyruvate) with 20 $\mathrm{mM}$ lactic acid or $\mathrm{HCl}$ for 24 hours and adjusted to a $\mathrm{pH}$ of 6.8, then OCR was measured as described above. c. A549, HOLO, and PARA cells were seeded in 6-well plates and cultured overnight in DMEM/F12 medium. Cells were then changed into starvation DMEM medium (no glucose, no glutamine, no pyruvate) containing $20 \mathrm{mM} \mathrm{L-lactic}$ acid or $\mathrm{HCl}$ and adjusted to $\mathrm{pH}$ 6.8. Cell number was determined after 6 days. ${ }^{*} P<0.01, * * \star P<0.001$ (one-way ANOVA with Tukey's multiple comparisons test). d. A549 and H358 cell lines were cultured in $100 \mathrm{~mm} \times 20 \mathrm{~mm}$ dishes containing DMEM/F12 medium to equilibrate overnight. Cells were then changed to DMEM medium without glucose containing $20 \mathrm{mM} \mathrm{L-lactic} \mathrm{acid} \mathrm{or} \mathrm{HCl}$ and adjusted to $\mathrm{pH}$ 6.8. After 5 days, cells were harvested and analyzed by FACS by staining with ALDEFLUOR $(\mathrm{ALDH})(\mathrm{n}=3)$ or GLDC $(\mathrm{n}=2)$ or SOX2 $(\mathrm{n}=2) .{ }^{*} P<0.05,{ }^{\star \star} P<0.01$ (two-tailed paired Student's t-test). Error bars represent mean \pm SD. 
Fig. 2

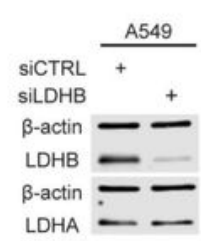

b

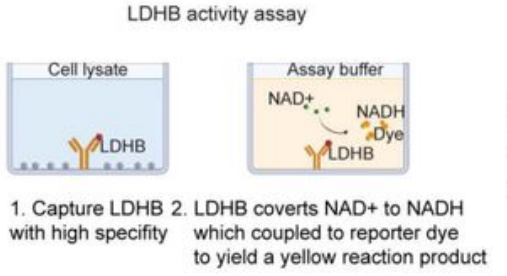

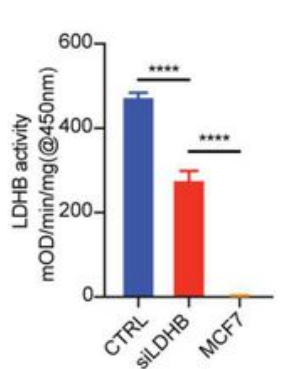

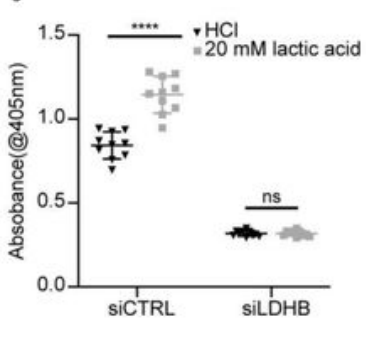

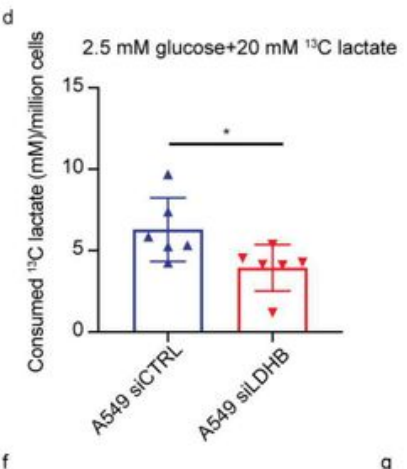

e
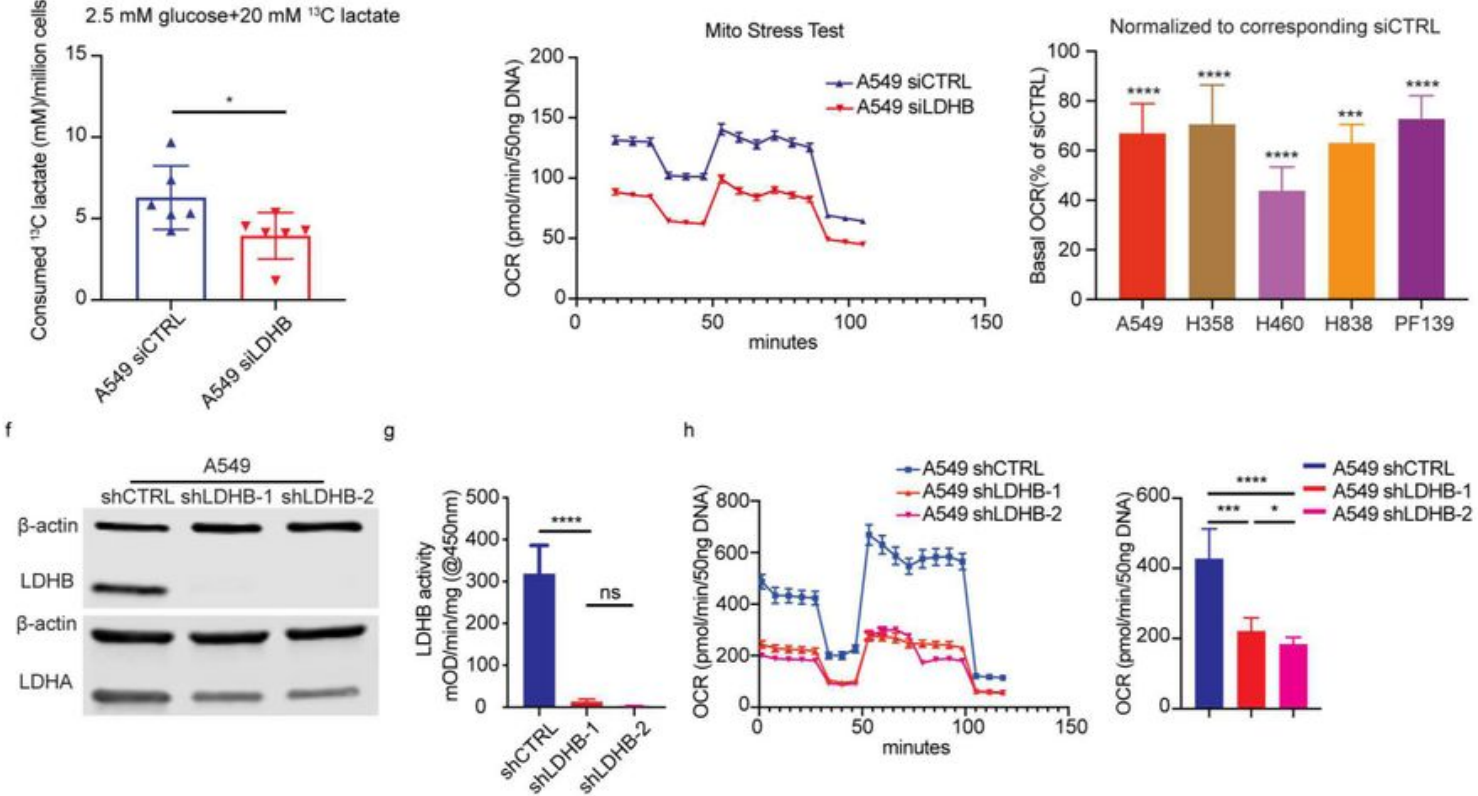

\section{Figure 2}

LDHB mediated lactate utilization is critical to OXPHOS.

a. Immunoblot analysis of A549 and H358 cells transfected with control siRNA (siCTRL) or LDHB-specific siRNA (siLDHB) (10 nM) using Lipofectamine 2000 after 48 hours. B-actin was used as the loading control. b. LDHB activity levels were determined in A549 cells 48 hours after transfection with siCTRL or 
siLDHB using an enzymatic colorimetric kit. The MCF7 cell line, expressing LDHA but not LDHB protein, was used as a negative control. Mean OD values were normalized to the number of cell lysates and reaction time. ${ }^{* \star \star *} P<0.0001$ (one-way ANOVA with Tukey's multiple comparisons test). c. siCTRL and siLDHB cells were plated at 3000 cells/well in 96-well plates for overnight culture. Cells then were changed into DMEM no glucose medium containing $20 \mathrm{mM} \mathrm{L-lactic} \mathrm{acid} \mathrm{or} \mathrm{HCl}$ and adjusted to $\mathrm{pH} 6.8$. After 4 days, cell viability was determined by Acid Phosphatase (APH) Assay. Absorbance was quantified in a Tecan Infinite ${ }^{\circledR}$ M1000 Microplate Reader at 405nm $(n=10)$. ${ }^{\star \star \star \star} P<0.0001$ (Ordinary two-way ANOVA). The error bars represent mean \pm SD. $d$. siCTRL and siLDHB cells were plated at $1 \times 10^{5}$ cells/well in 6-well plates for overnight culture. Cells then were changed into DMEM medium containing $2.5 \mathrm{mM}$

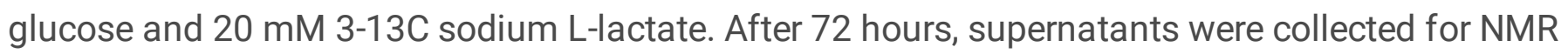
analysis. The measurement was previously described (71-74). For each sample, the ${ }^{13} \mathrm{C}$ lactate peak of interest was integrated. The absolute integral was then converted to an $\mathrm{mM}$ value using the integral of the initial condition with a known concentration of $20 \mathrm{mM}$ lactate. Lactate consumption values were normalized to the corresponding cell number and then used for statistical analysis $(n=6)$. ${ }^{*}<0.05$ (twotailed unpaired Student's t-test). Data are shown with mean \pm SD. e. Real-time oxygen consumption rate (OCR) and extracellular acidification rate (ECAR) were measured after 48 hours of transfection with siCTRL or siLDHB using the Seahorse Xfe96 analyzer. The procedure corresponds to the description in Fig. 1. The basal OCR and ECAR were used to perform the statistical analysis. The normalized OCR and ECAR were plotted as bar graphs $(n=11-24)$. The error bars represent mean \pm SEM. $\mathbf{f - g}$. Immunoblot analysis of A549 shCTRL (scramble control) and shLDHB clones. B-Actin was used as a loading control. LDHB activity levels were determined as described above. h. Real-time oxygen consumption rate (OCR) of A549 shCTRL and shLDHB clones were measured using the Seahorse Xfe96 analyzer $(n=10)$. The error bar was represented with mean \pm SEM (left panel) and mean \pm SD (right panel). ${ }^{\star} P<0.05,{ }^{\star} * \star P<0.001$, $\star \star \star \star P<0.0001$ (Ordinary one-way ANOVA). 

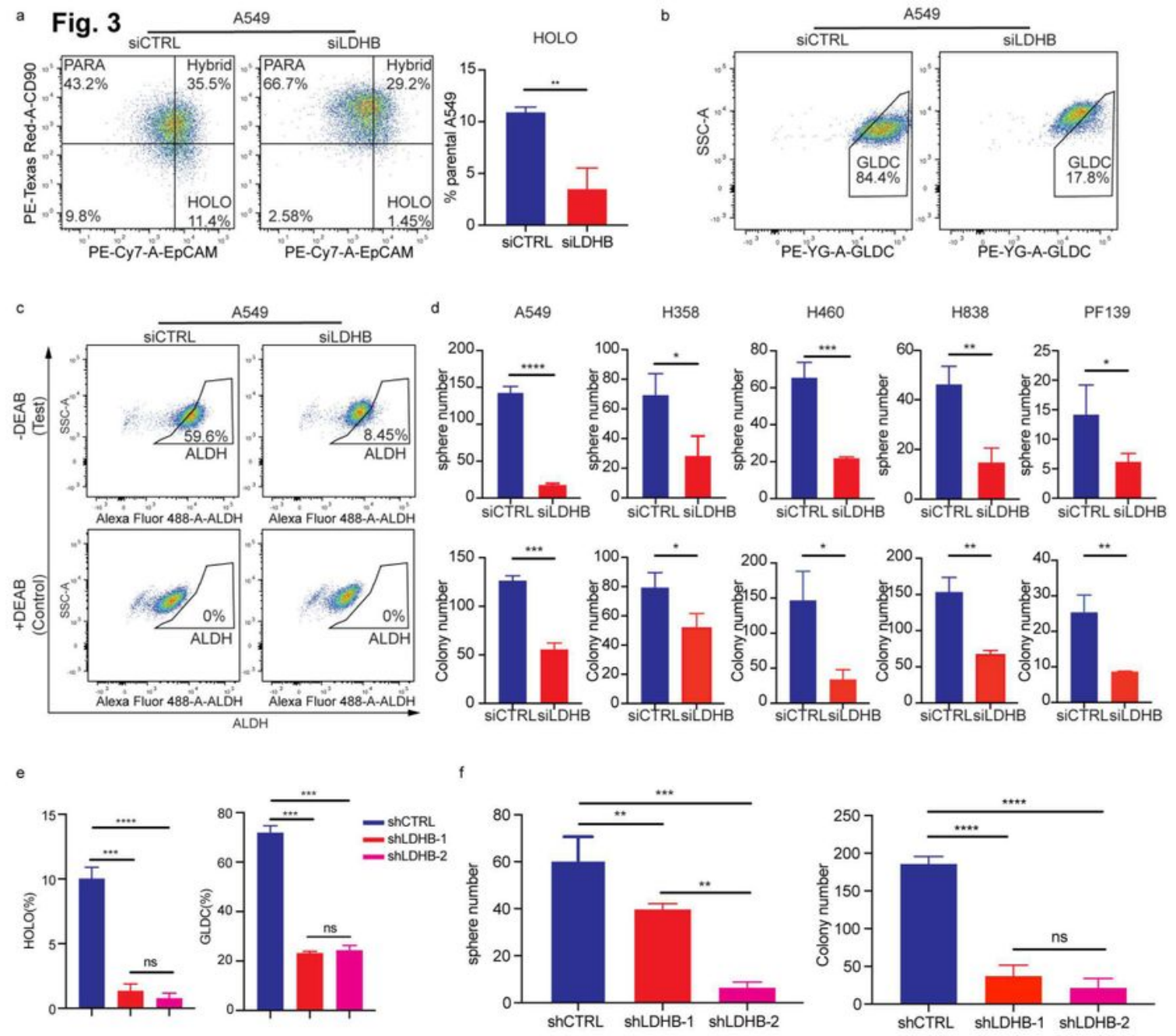

9
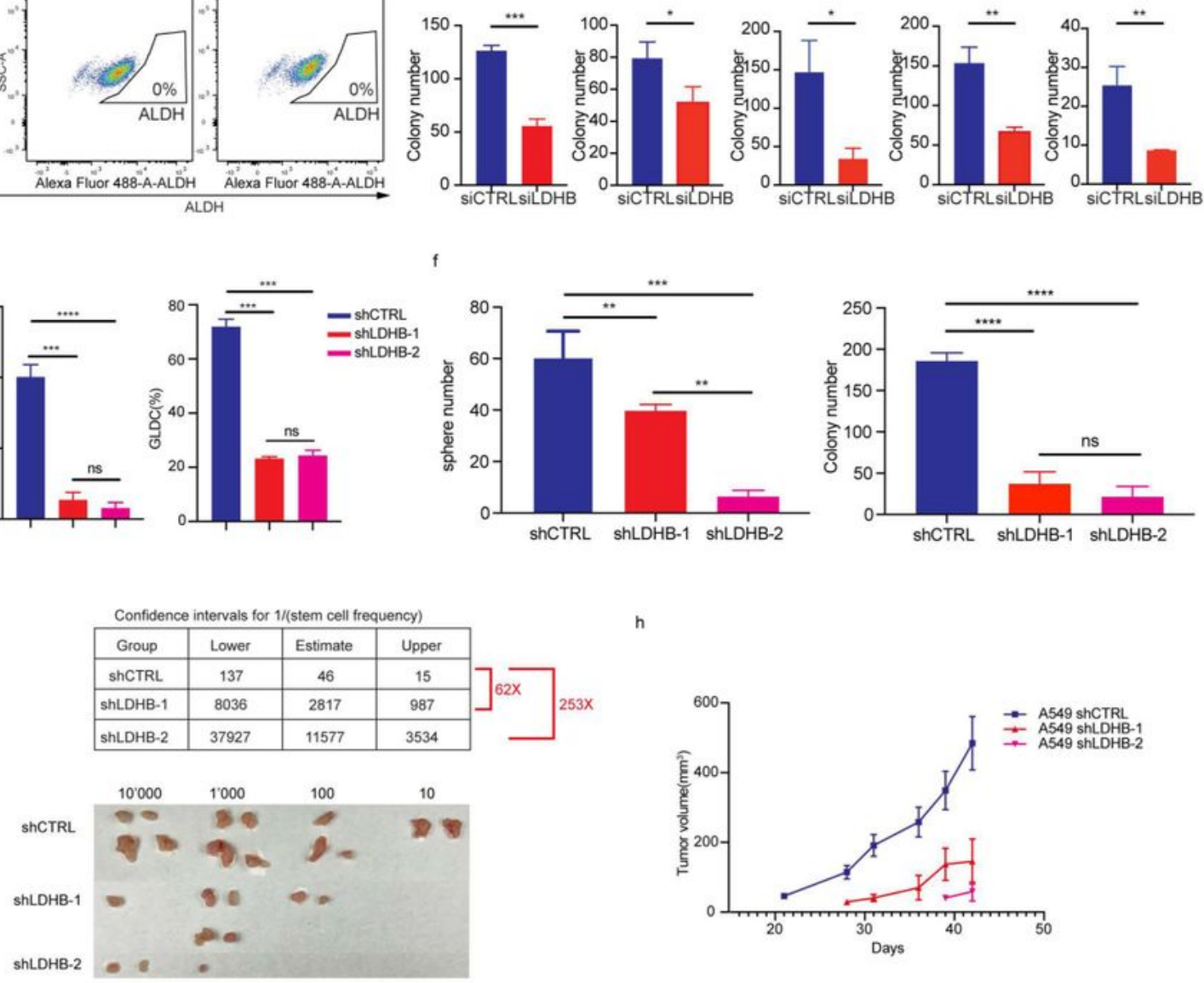

Figure 3

LDHB inhibition results in reduced survival of TICs, tumor initiating capacity and growth.

a. Analysis of HOLO and PARA cells by flow cytometer using EPCAM and CD90 co-staining after 48 hours of transfection with siCTRL or siLDHB. HOLO and PARA cells are represented as $\mathrm{EpCAM}^{+} / \mathrm{CD}^{-} 0^{-}$and EpCAM $/ \mathrm{CD}^{2} 0^{+}$, respectively. b-c. Analysis of TICs population by flow cytometer using staining with 
markers for TICs, e.g., ALDH after 48 hours of transfection with siCTRL or siLDHB (n=2). D. 500 siCTRL or siLDHB cells were seeded in Nunclon Sphera 6-well plates and cultured with 3D CnT culture medium or 2D DMEM/F12 medium after transfection with siCTRL or siLDHB. Images were taken after 7-14 days. For the sphere formation assay, spheres were counted under the microscope, and for the colony formation assay, colonies were counted with Fiji $(\mathrm{n}=3)$ after 7-21 days. $n s$ no significant difference, ${ }^{\star} P<0.05$, $* \star P<$ 0.01 , $* \star \star P<0.001$ (two-tailed unpaired Student's t-test). Data are shown with mean \pm SD. eff. Analysis of HOLO and PARA cells, and GLDC positive population by flow cytometer as same as described above ( $\mathrm{n}=2-$ 3). ${ }^{*} P<0.05,{ }^{*} P<0.01,{ }^{*} * * P 0.001,{ }^{*} * \star * P<0.0001$ (Ordinary one-way ANOVA). The error bars are represented with mean \pm SD. $\mathbf{g}$. The stem cell frequency was calculated based on extreme limited dilution analysis (details described in the methods section). Images of tumor explants 6 weeks after injection of different cell numbers of shCTRL and shLDHB clones (10, 100, 1,000, 10,000 cells). h. Growth curves were determined based on tumor volume at different time points. 
a Fig. 4

A549CTRvsKD DOWN
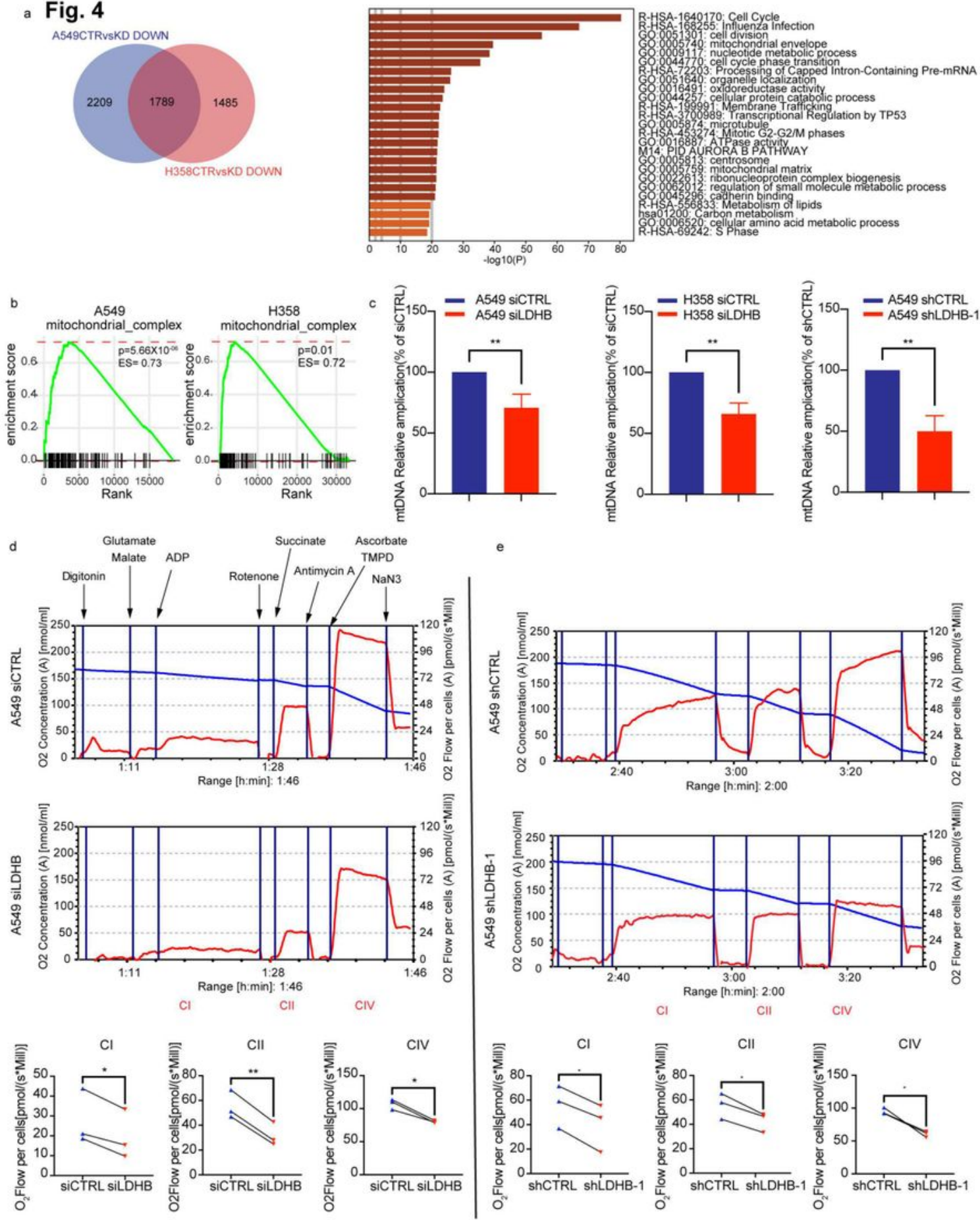

Figure 4

OXPHOS deficiency induced by LDHB inhibition is related to persistent mtDNA damage.

a. Venn diagram showing the number of genes down-regulated in A549 and H358 after LDHB silencing. Comprehensive pathway analysis showing top 25 down-regulated pathways or gene ontology terms in A549 and H358 after LDHB silencing. b. Relative RNA expression-based enrichment of Mitochondrial 
Respiratory Chain Complexes in response to mitochondrial complex activity in siCTRL compared to siLDHB. The $y$-axis represents the enrichment value, and the $x$-axis the rank of differential expression for all genes, with group-specific genes indicated by vertical black lines. Rank positions to the left indicate increased expression in siCTRL. c. The mtDNA copy number and mtDNA damage were determined by LORD-Q analysis on isolated DNA from SiCTRL and siLDHB after 48 hours of transfection or shCTRL and shLDHB cells $(\mathrm{n}=3-8) .{ }^{*} P<0.05,{ }^{*} P<0.01,{ }^{*} * * P<0.001,{ }^{*} * \star * P<0.0001$ (two-tailed paired Student's ttest). The error bars represent mean \pm SD. d-e. Maximal ADP-stimulated respiration was quantified by OROBOROS to evaluate mitochondrial respiration complex I (CI), II (CII), IV (CIV) activity in A549 siCTRL and A548 siLDHB after 48 hours of transfection or A549 shCTRL and shLDHB cells after supplementation with digitonin (permeabilization), glutamate and malate (activation of complex I activity), rotenone (complex I inhibitor), succinate (activation of complex II activity), antimycin A (complex II inhibitor), ascorbate/TMPD (activation of complex IV activity), NaN3 (complex IV inhibitor) sequentially. The respiration rate is expressed in pmol/ (sec $x$ million cells) $(\mathrm{n}=3) .{ }^{\star} P<0.05,{ }^{*} P<0.01$ (two-tailed paired Student's t-test). The error bars represent mean \pm SD.

\section{Figure 5}

LDHB inhibition downregulates mitochondrial-dependent pyrimidine and purine synthesis pathways.

a-b. Venn diagram showing the number of metabolites reduced in A549 and H358 after LDHB silencing. E. Balloon plot showing metabolite enrichment analysis (MSEA) of reduced metabolites in A549 and H358 after LDHB silencing. The top 10 dysregulated metabolic pathways are shown. The size and color of the balloon indicate the enrichment ratio and $P$-value, respectively. Heatmap showing the metabolomic comparison of siCTRL and siLDHB cells. Six replicates (including three biological replicates with two technical replicates) are shown as separate columns for each cell type. Log2 of the ratio between the metabolite intensity of each sample to the average intensity of all samples. c. For proliferation and sphere formation ability, rescue experiments were performed with the addition of the following nucleotide precursors: $100 \mu \mathrm{M}$ hypoxanthine, $100 \mu \mathrm{M}$ adenine, $400 \mu \mathrm{M}$ uridine. The number of cells and spheres was counted after 5 and 7 days with nucleotide precursor rescue. Ns no significant difference, ${ }^{\star} P<0.05, \star \star P<$

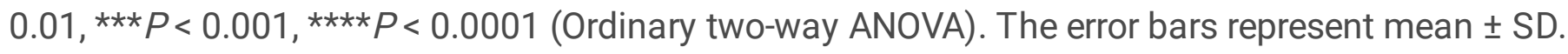

\section{Figure 6}

Depletion of LDHB results in decreased tumorigenesis and delayed tumor growth in a genetically engineered mouse model of NSCLC.

a. Schematic representation of the lung tumor induction experiment in LDHB knockout and wild-type

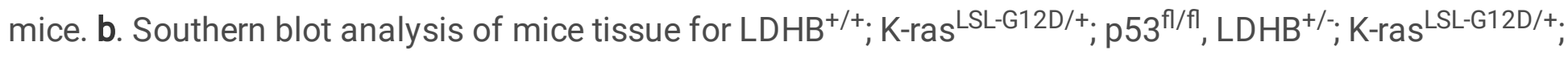




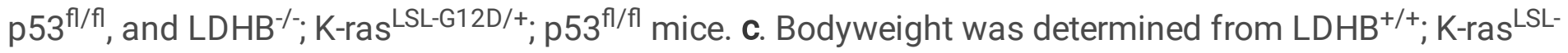

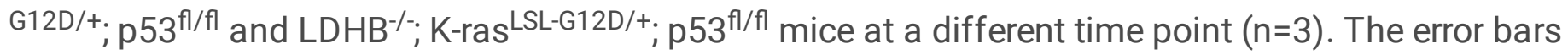

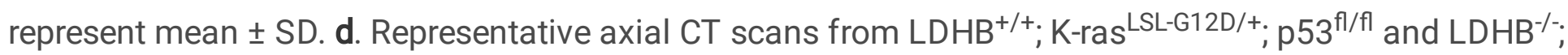
K-ras ${ }^{\mathrm{LSL}-\mathrm{G} 12 \mathrm{D} /+} ; \mathrm{p} 53^{\mathrm{f} / \mathrm{fl}}$ mice after intratracheal instillation with AAV-Cre virus at different time points. e. 3D

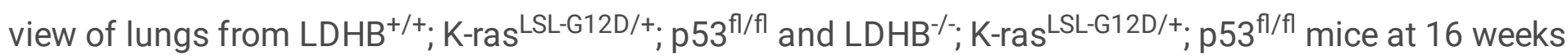

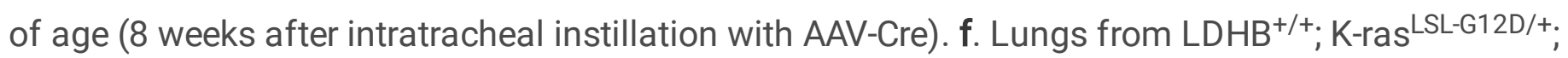
$\mathrm{p} 53^{\mathrm{fl} / \mathrm{fl}}$ and $\mathrm{LDHB}^{-/-} ; \mathrm{K}$-ras ${ }^{\mathrm{LSL}-\mathrm{G} 12 \mathrm{D} /+} ; \mathrm{p} 53^{\mathrm{fl} / \mathrm{fl}}$ mice were harvested and weighed after 8 weeks of intratracheal instillation with AAV-Cre virus $(n=3)$. A two-tailed paired Student's t-test was used for statistical analysis. The error bars represent mean \pm SD. $\mathbf{g}$. The representative HE images are from the mice sacrificed above.

\section{Figure 7}

Lactate supplementation increases survival of patient-derived tumor tissue ex vivo.

a-b. Schematic representation of an experiment where PCTS was cultured in $10 \mathrm{mM}$ glucose DMEM medium with or without $20 \mathrm{mM}$ sodium lactate. $\mathbf{c}$. Analysis of cell viability of fresh precision tissue sections (PCTS) after addition of $20 \mathrm{mM}$ sodium lactate in DMEM medium containing $2.5 \mathrm{mM}$ glucose or $10 \mathrm{mM}$ glucose after 3 days by Calcein AM staining (green) for live cells, EthD-1 staining (red) for dead cells and HOECHST (blue) for DNA. The images were taken with a Zeiss LSM 880 confocal microscope and analyzed by Fiji. The Live/Dead ratio is presented $(n=2) .{ }^{*} P<0.05$ (two-tailed paired Student's t-test).

\section{Supplementary Files}

This is a list of supplementary files associated with this preprint. Click to download.

- SupplementtaryExcelfileGeneExpression.xIsx

- SupplementaryExcelfileMetabolomicsData.xlsx

- S1.jpg

- S2.jpg

- S3.png

- $54 . j p g$

- S5.png

- S6.jpg

- 57.jpg

- Tables.pdf 